Pacific Journal of Mathematics

RATIONAL HOMOTOPY THEORY OF FIBRATIONS 


\title{
RATIONAL HOMOTOPY THEORY OF FIBRATIONS
}

\author{
Flavio E. A. DA Silveira
}

\begin{abstract}
Let $Y$ be a space and $A$ a differential graded algebra over the field $Q$ of rationals corresponding in Sullivan's theory to the rational homotopy type of $Y$. Then to the rational homotopy type of a fibration over $Y$ equipped with a given cross-section corresponds a differential graded Lie algebra $L$ over $A$, free as an $A$-module. The differential graded Lie algebra $\mathbf{Q} \otimes_{A} L$ corresponds in Quillen's theory to the rational homotopy type of the fibre of the fibration. Furthermore, by restriction of scalars, $L$ can be considered as a differential graded Lie algebra over $Q$. Then it contains a differential graded Lie sub-algebra over $\mathbf{Q}$ which corresponds to the rational homotopy type of the space of cross-sections which are homotopic to the given cross-section. Some examples illustrate this result.
\end{abstract}

\section{TABLE OF CONTENTS}

1. Introduction AND Statement OF THE MAin Results

2. $F^{2}$-Free Modules AND SpeCtral Sequences. Description of the spectral sequence naturally associated to a $F^{2}$-free module

3. Duality. Proof of the equivalence between (2) and (3) in Theorem 1

4. QUILLEN's Functors $\varrho_{A}$ AND $\mathcal{C}_{A}$. Extension of Quillen's functors $\mathcal{L}$ and $C_{\text {to }}$ DG-coalgebras and DG-Lie algebras over a DG-algebra. Proof of the equivalence between (3) and (4) in Theorem 1

5. The MOdel $L_{+}$FOR THE SPACE OF CROSS-Sections. Proof of Theorem 2 ................... 17

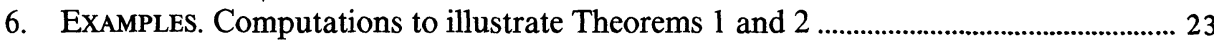

\section{Introduction and statement of the main results.}

Rational homotopy theory. Quillen [11] and Sullivan [12] established the equivalence of the following four categories:

(1) the rational category of simply connected spaces with rational homology of finite dimension in each degree;

(2) the rational category of DG-algebras $A$ (differential graded associative and commutative algebras over Q) such that $\operatorname{dim} A^{p}<\infty$ for each $p, A^{p}=0$ if $p<0$ or $p=1$, and $A^{0}=\mathbf{Q}$;

(3) the rational category of DG-coalgebras $C$ (differential graded co-associative and co-commutative coalgebras over $\mathbf{Q}$ ) such that $\operatorname{dim} C_{p}<$ $\infty$ for each $p, C_{p}=0$ if $p<0$ or $p=1$ and $C_{0}=\mathbf{Q}$;

(4) the rational category of DG-Lie algebras (differential graded Lie algebras over Q) such that $\operatorname{dim} L_{p}<\infty$ for each $p$ and $L_{p}=0$ if $p \leq 0$. 
By "rational category" we mean the category localized relatively to the weak equivalences, i.e. to those maps which induce an isomorphism on (the rational) homology.

Sullivan demonstrated the equivalence between (1) and (2) more generally for nilpotent spaces and for DG-algebras with a free minimal nilpotent model. In fact, recently Neisendorfer [10] has shown that the dictionary described above generalizes to nilpotent spaces with rational homology of finite dimension in each degree. The corresponding DG-Lie algebras do not necessarily vanish in degree zero but are supposed to have a rational homology with a nilpotent completion.

Quillen proved the equivalence between (1), (3) and (4). The equivalence between (1) and (4) is such that if $L$ is a DG-Lie algebra which is a model for a space $X$, then there is a graded Lie algebra isomorphism $H_{*}(L)=\pi_{*}(\Omega X) \otimes_{\mathbf{Z}} \mathbf{Q}$, where $H_{*}(L)$ has the induced bracket and $\pi_{*}(\Omega X)$ is equipped with the Whitehead product. We shall return to the equivalence between (3) and (4) in \$4.

We shall say that an object in (2), (3) or (4) corresponding to a space $X$ in (1) is a model for $X$.

The case of a fibration. Let $Y$ be a space and $A$ a DG-algebra which is a model for $Y$. It is a consequence [5] of Sullivan's theory that the two following categories are equivalent:

$(1)^{\prime}$ the rational category of fibrations $p: X \rightarrow Y$ (satisfying conditions analogous to those satisfied by spaces in (1)); and

$(2)^{\prime}$ the rational category of DG-algebras $E$ such that, as algebras, $E=A \otimes F$, where $F$ is a DG-algebra (and satisfying some technical conditions).

Actually, we have two results which, roughly speaking, can be stated as follows.

1. The Quillen-Sullivan dictionary for the rational homotopy type of spaces generalizes, under certain conditions, to a dictionary for the rational homotopy type of fibrations over a space $Y$ equipped with a given cross-section. In the latter dictionary, the DG-algebra A, which is a model for $Y$, substitutes the field $\mathbf{Q}$ in the former dictionary, so that the algebraic objects involved are DG-algebras, DG-coalgebras and DG-Lie algebras over A, free as A-modules.

The fact that there is a given cross-section corresponds, in the case of the DG-algebras or the DG-coalgebras over $A$, to the fact that a DG-algebra $A$-augmentation or a DG-coalgebra $A$-augmentation is given. As to 
the DG-Lie algebras over $A$, however, their very structure depends on the given cross-section. Moreover, the DG-Lie algebras over $A$ carry additional information, as asserted below.

2. A DG-Lie algebra over $A$ in 1 , considered as a DG-Lie algebra over $\mathbf{Q}$, contains a canonical DG-Lie sub-algebra, which corresponds in the Quillen-Sullivan dictionary to the space of cross-sections homotopic to the given cross-section.

In $\S 6$ we give concrete applications of 2 .

To state results 1 and 2 with precision we need the following definitions and conventions.

Definitions and conventions. Let $A$ be a DG-algebra over a field $K$. A DG-module over $A$ is a differential graded vector space $M$ with an $A$-module structure $m: A \otimes M \rightarrow M$ such that $m(1 \otimes x)=x$ and $d m=$ $m(d \otimes \mathrm{id}+\mathrm{id} \otimes d)$.

Given a DG-module $E$ over $A$, one defines a structure on $E$ of $D G$-algebra over $A$ in the same way as one defines a DG-algebra, replacing the field of scalars by the DG-algebra $A$ in the structure maps (multiplication and unity).

In the same fashion one defines a $D G$-coalgebra $\mathscr{X}$ over $A$ and a DG-Lie algebra L over $A$.

Also one defines an augmentation of $E$ (respectively $\mathcal{X}$ ) to be a DG-algebra (DG-coalgebra) map $E \rightarrow A(A \rightarrow \mathcal{X})$ over $A$ such that $A \stackrel{\text { unity }}{\rightarrow} E \rightarrow A(A \rightarrow \mathcal{X} \stackrel{\text { counity }}{\rightarrow} A)$ is the identity.

Note that if $A$ is augmented and $K$ has the structure of an $A$-module induced by the augmentation, then $K \otimes_{A} E, K \otimes_{A} \mathcal{X}$ and $K \otimes_{A} L$ are naturally equipped with a structure of DG-algebra, DG-coalgebra and DG-Lie algebra, respectively.

Let $\mathcal{E}$ be one among the three objects $E, \mathscr{X}$ or $L$. As a DG-module over $A$ it is equipped with the natural filtration given by $F^{p} \mathcal{E}=\left(F^{p} A\right) \mathcal{E}$, where $F^{p} A$ is the ideal of those elements of $A$ with degree $\geq p$. Assume $A$ is augmented and $\mathscr{E}$ is a free $A$-module. Then there exists an $A$-module isomorphism $\mathcal{E} \approx A \otimes \mathscr{F}$, where $\mathscr{F}=K \otimes_{A} \mathcal{E}$, which does not necessarily preserve the DG-algebra, DG-coalgebra, DG-Lie-algebra structure over $A$. We shall say that $\mathcal{E}$ is a $F^{2}$-free $D G$-algebra over $A$ (respectively, $F^{2}$-free $D G$-coalgebra over $A, F^{2}$-free $D G$-Lie algebra over $A$ ) if there exist an 
$A$-module isomorphism such that if $\mathcal{E}$ is identified to $A \otimes \mathscr{F}$ then, for every $x, y \in \mathcal{F}$,

$$
d_{\mathscr{E}}(1 \otimes x)-1 \otimes d_{\mathscr{F}} x \in F^{2} \mathcal{E} \quad \text { and } \quad(1 \otimes x)(1 \otimes y)-1 \otimes x y \in F^{2} \mathcal{E}
$$

(respectively the same condition on the differentials and $\Delta_{\mathscr{G}}(1 \otimes x)-1 \otimes$ $\left.\Delta_{\mathscr{F}} x \in F^{2} \mathcal{E} \otimes_{A} F^{2} \mathcal{E},[1 \otimes x, 1 \otimes y]-1 \otimes[x, y] \in F^{2} \mathcal{E}\right)$.

In this paper we denote by $K$ a field of characteristic zero. Most of the time $K$ stands actually for $\mathbf{Q}$, the field of rationals. (This is the case whenever the algebraic objects are related to geometric objects.)

All graded objects in this paper have a structure of graded vector space over $K$ and are graded by negative and positive integers. We shall adopt the convention that if $V$ is a graded vector space, then $V^{p}=V_{-p}$ for any integer $p$. Unless otherwise stated we shall assume all DG-algebras to be upper graded and to vanish in negative degrees, and all DG-coalgebras and DG-Lie algebras to be lower graded and to vanish in negative degrees.

Note that while in a DG-algebra $E$ over $A$ the $A$-module structure is such that if $a \in A^{p}$ and $x \in E^{q}$, then $a x \in E^{p+q}$; in a DG-coalgebra $\mathcal{X}$ over $A$ or in a DG-Lie algebra $L$ over $A$ the $A$-module structure is such that if $a \in A^{p}$ and $x \in \mathcal{X}_{q}$ or $L_{q}$, then $a x \in \mathcal{X}_{q-p}$ or $L_{q-p}$. DG-algebras over $A$ appear naturally to be positively graded, whereas DG-coalgebras or DG-Lie algebras over $A$ have in general non-trivial elements in negative degrees.

We shall denote by $A(V)=A\left(x_{1}, x_{2}, \ldots, x_{n}, \ldots\right)$ the free associative, commutative and graded algebra over a DG-algebra $A$ generated by a graded vector space $V$ with base $x_{1}, x_{2}, \ldots, x_{n}, \ldots$, i.e. $A(V)=A \otimes \mathbf{Q}(V)$ and $\mathbf{Q}(V)=\mathbf{Q}\left(x_{1}, x_{2}, \ldots, x_{n}, \ldots\right)$ is the symmetric algebra on $V$ (which is isomorphic to the tensor product of the polynomial algebra on the subspace of $V$ spanned by the elements of even degree by the exterior algebra on the subspace spanned by the elements of odd degree).

We shall denote by $S_{A}(M)$ the symmetric coalgebra over a DG-algebra $A$ on a module $M$ over $A$. It is the sub-coalgebra over $A$ of the tensor coalgebra over $A$ on $M$ defined by taking in $\otimes_{A}^{n} M=M \otimes_{A} M \otimes_{A}$ $\cdots \otimes_{A} M$ the sub- $A$-module of invariant elements under the action of the symmetric group. If $A=\mathbf{Q}, S_{\mathbf{Q}}(M)=S(M)$ is the usual symmetric coalgebra on the graded vector space $M$.

\section{A Relative Version for the Rational Homotopy TheOry.}

THEOREM 1. Let $Y$ be a nilpotent space and assume $A$ to be $a$ $D G$-algebra which is a model for $Y$ and such that $A^{p}=0$ if $p$ is large enough. Then the following four categories are equivalent: 
(1) the rational category of fibrations $p: X \rightarrow Y$ equipped with a given cross-section $s: Y \rightarrow X$ (the maps being those which preserve the cross-sections) such that $p$ is induced from a fibration with simply connected base and such that the fibre of $p$ is simply connected and has a rational homology of finite dimension in each degree;

(2) the rational category of augmented $F^{2}$-free DG-algebras $E$ over $A$ (the maps being those which preserve the augmentations) and such that $\left(\mathbf{Q} \otimes_{A} E\right)^{p}=0$ if $p<0$ or $p=1,\left(\mathbf{Q} \otimes_{A} E\right)^{0}=\mathbf{Q}$ and $\operatorname{dim}\left(\mathbf{Q} \otimes_{A} E\right)^{p}<\infty$ for every $p$;

(3) the rational category of augmented $F^{2}$-free DG-coalgebras $\mathcal{X}$ over $A$ (the maps being those which preserve the augmentations) and such that $\left(\mathbf{Q} \otimes_{A} \mathfrak{X}\right)_{p}=0$ if $p<0$ or $p=1,\left(\mathbf{Q} \otimes_{A} \mathfrak{X}\right)_{0}=\mathbf{Q}$ and $\operatorname{dim}\left(\mathbf{Q} \otimes_{A} \mathfrak{X}\right)_{p}<$ $\infty$ for every $p$;

(4) the rational category of $F^{2}$-free DG-Lie algebras $L$ over $A$ such that $\left(\mathbf{Q} \otimes_{A} L\right)_{p}=0$ if $p \leq 0$ and $\operatorname{dim}\left(\mathbf{Q} \otimes_{A} L\right)_{p}<\infty$ for every $p$.

Furthermore, the equivalences are functorial relative to the changes of base.

By change of base in (2), (3) or (4) we mean the following: if $\mathcal{E}$ is an $A$-module and $f: A \rightarrow A^{\prime}$ is a DG-algebra map, then, considering $A^{\prime}$ equipped with the structure of an $A$-module induced by $f, A^{\prime} \otimes_{A} \delta$ is the $A^{\prime}$-module obtained from $\mathcal{E}$ by the change of base $f$.

In particular, Theorem 1 is such that if $Y$ is a point and $A=\mathbf{Q}$, the equivalences correspond to the dictionary for the homotopy type of a simply connected space.

Note that again by "rational category" we mean the category localized relatively to the weak equivalences over $Y$ or $A$, i.e. to those maps over $Y$ or $A$ which induce an isomorphism on (the rational) homology.

We shall say that an object in (2)-(4) corresponding to a fibration $p$ : $X \rightarrow Y$ in (1) is a model over $A$ for $p$.

As mentioned above, the equivalence between (1) and (2) is much more general and is a consequence of Sullivan's theory of the free minimal model. It is functorial relative to the changes of base, and if $E$ is a DG-algebra over $A$ which is a model over $A$ for a fibration $p: X \rightarrow Y$, then $\mathbf{Q} \otimes_{A} E$ is a DG-algebra which is a model for the fiber of $p$. It is also easily verified that to a cross-section $s$ of $p$ corresponds an augmentation of the free minimal model of $p$ over $A$ and conversely.

The proof of Theorem 1 is therefore entirely algebraic and consists in describing the equivalences between (2)-(4). 
A Model for the Space of Cross-sections Homotopic to a Given CRoss-SECTION.

The following theorem stresses the interest of Theorem 1.

THEOREM 2. Let $Y$ be a nilpotent space and assume $A$ is a DG-algebra which is a model for $Y$ such that $A^{0}=\mathbf{Q}$ and $\operatorname{dim} A<\infty$ as a $\mathbf{Q}$-vector space.

Let $p: X \rightarrow Y$ be a fibration with a given cross-section $s: Y \rightarrow X$ verifying condition (1) in Theorem 1 and let L be a DG-Lie algebra over $A$ verifying condition (4) in Theorem 1 and which is a model over $A$ for $p$.

By restriction of scalars consider $L$ as a DG-Lie algebra over $\mathbf{Q}$. Let $L_{+}$be the DG-Lie sub-algebra defined by $\left(L_{+}\right)_{p}=L_{p}$ if $p>0,\left(L_{+}\right)_{0}=$ $\operatorname{ker}\left(d: L_{0} \rightarrow L_{-1}\right)$ and $\left(L_{+}\right)_{p}=0$ if $p<0$.

Then $L_{+}$is a model for the connected component of $s$ in the space of cross-sections of $p$.

The construction of a model for the space of cross-sections homotopic to a given cross-section has been suggested by Thom [13] and Sullivan, and the fact that this construction actually yields a model has been proved by Haefliger [8]. This is the crucial result we use to prove Theorem 2. Note that if $Y$ is a finite-dimensional nilpotent space with finite-dimensional homotopy groups, then there always exists a finite-dimensional DG-algebra $A$ which is a model for $Y$ [6] (page 517).

The following two corollaries are immediate consequences of Theorem 2.

COROLlARY. Keep the same hypotheses and notation as in Theorem 2 and assume that in the fibration $p$ the dimension of the base $Y$ is lower than the connectivity of the fiber. Let $\Gamma_{p}$ be the space of cross-sections of $p$. Then $\pi_{*}\left(\Gamma_{p}\right) \otimes_{\mathbf{Z}} \mathbf{Q}$ is naturally equipped with an $H^{*}(Y, \mathbf{Q})$-module structure.

Proof. Choose $L$ to be positively graded. Then $H(L)$ is naturally a $H(A)$-module.

COROLlaRY. Let $A$ be a graded algebra (with trivial differential) such that $A^{0}=\mathbf{Q}, A^{q}=0$ for $q<0$ and $\operatorname{dim} A<\infty$ as a $\mathbf{Q}$-vector space. Let $L$ be a Lie algebra over $A$ (also with trivial differential) free as an A-module and assume $L$ is positively graded and such that $\operatorname{dim}\left(\mathbf{Q} \otimes_{A} L\right)_{q}<\infty$ for 
every $q$ and $\left(\mathbf{Q} \otimes_{A} L\right)_{0}=0$. Then there exists a fibration $p: X \rightarrow Y$ such that, as Lie algebras over $\mathbf{Q}, L \cong \pi_{*}\left(\Omega \Gamma_{p}\right) \otimes_{\mathbf{Z}} \mathbf{Q}$, where $\Omega \Gamma_{p}$ is the loop space on the space $\Gamma_{p}$ of cross-sections of $p$.

Proof. Choose $p: X \rightarrow Y$ such that $L$ is a model for $p$ over $A$ and note that, over $\mathbf{Q}, L=L_{+}$.

2. $F^{2}$-free modules and spectral sequence. This section is inspired by the lemma on page 509 in [6].

$F^{2}$-free modules. Let $A$ be an augmented DG-algebra and $M$ a DG-module over $A$. As in $\S 1$ we say $M$ is a $F^{2}$-free module over $A$ if $M$ is a free $A$-module and if there exists an $A$-module isomorphism $\varphi: A \otimes\left(K \otimes_{A} M\right) \stackrel{\approx}{\rightarrow} M$ such that $d \varphi(1 \otimes x)-\varphi\left(1 \otimes d^{\prime} x\right) \in F^{2} M$ for every $x \in K \otimes_{A} M$, where $d^{\prime}$ is the differential induced on $K \otimes_{A} M$ by the differential $d$ on $M, K$ is equipped with the $A$-module structure induced by the augmentation of $A$, and, by definition, $F^{p} M=\left(F^{p} A\right) M, F^{p} A=$ $\oplus_{q \geq p} A^{q}$.

Spectral sequence. The following lemma follows from the definitions.

(2.1) LeMmA. Let $A$ and $M$ be as above and assume that either $M^{p}=0$ if $p<0$ or there is an integer $n \geq 0$ such that $A^{p}=0$ for $p>n$ and $\left(K \otimes_{A} M\right)_{r}=0$ whenever $r<0$. Assume $M$ is $F^{2}$-free over $A$ and choose an $A$-module isomorphism $\varphi: A \otimes\left(K \otimes_{A} M\right) \underset{\sim}{\rightarrow} M$ such that $d \varphi(1 \otimes x)-$ $\varphi\left(1 \otimes d^{\prime} x\right) \in F^{2} M$ for every $x \in K \otimes_{A} M$. Then the spectral sequence associated to the natural filtration $F^{p} M$ converges to $H(M)$ and $\varphi$ induces an isomorphism $E_{2} \approx H(A) \otimes H\left(K \otimes_{A} M\right)$.

The assumptions on $A$ and $M$ are such that the spectral sequence is like a first quadrant spectral sequence.

Comparison of spectral sequences. Let $A$ and $A^{\prime}$ be two augmented DG-algebras and let $f: A \rightarrow A^{\prime}$ be a DG-algebra map. Let $M$ be a DG-module over $A, M^{\prime}$ a DG-module over $A^{\prime}$, and $\alpha: M \rightarrow M^{\prime}$ a map of modules over $f$ (i.e. an $A$-module map where the $A$-module structure on $M^{\prime}$ is induced by $f$ ). Let $\alpha^{K}: K \otimes_{A} M \rightarrow K \otimes_{A^{\prime}} M^{\prime}$ be the induced map of DG-vector spaces (equipped with the induced differentials).

(2.2) Corollary. Assume $M$ and $M^{\prime}$ are $F^{2}$-free over $A$ and $A^{\prime}$, respectively, and either $M^{p}=M^{\prime p}=0$ if $p<0$ or there are two integers 
$n, m \geq 0$ such that $A^{p}=A^{\prime q}=0$ whenever $p>n, q>m$ and $\left(K \otimes_{A} M\right)_{r}$ $=\left(K \otimes_{A^{\prime}} M^{\prime}\right)_{r}=0$ if $r<0$. Assume $f$ is a weak equivalence. Then $\alpha$ is a weak equivalence if and only if $\alpha^{K}$ is a weak equivalence.

The proof is straightforward using (2.1) and Moore's comparison theorem [2]. As a consequence of (2.1) and (2.2) we have the following.

(2.3) COROLlary. With the same notations and hypotheses as in (2.2), assume, furthermore, there are $N, N^{\prime}$ and $\beta: N \rightarrow N^{\prime}$ like $M, M^{\prime}$ and $\alpha$, respectively, and also satisfying conditions as in (2.2). Assume $f, \alpha$ and $\beta$ are weak equivalences. Then the map $\alpha \otimes_{f} \beta: M \otimes_{A} N \rightarrow M^{\prime} \otimes_{A^{\prime}} N^{\prime}$ of DG-modules over $f$ is a weak equivalence.

The functor $\operatorname{Hom}_{A}(, A)$. Let $A$ be a DG-algebra and $M$ and $N$ two DG-modules over $A$. Recall that $\operatorname{Hom}(M, N)$, graded by $\operatorname{Hom}(M, N)_{p}=$ $\Pi_{r} \operatorname{Hom}\left(M_{r}, N_{p+r}\right)$ with the differential defined by $(d f)(m)=d_{N}(f(m))$ $-(-1)^{\operatorname{deg} f} f\left(d_{M} m\right)$, is a DG-module over $A$, where $a \cdot f$ is defined by $(a \cdot f)(m)=a \cdot f(m), a \in A, f \in \operatorname{Hom}(M, N), m \in M$.

As usual we shall denote by $\operatorname{Hom}_{A}(M, N)$ the sub-DG-module over $A$ of $\operatorname{Hom}(M, N)$ consisting of all $f \in \operatorname{Hom}(M, N)_{p}$ such that for every $a \in A^{q}$ and every $m \in M, f(a m)=(-1)^{p q}(a \cdot f)(m)$.

It is easy to verify that, for every DG-vector space $V$,

$$
\operatorname{Hom}_{A}(A \otimes V, A)=\operatorname{Hom}(V, A),
$$

and, if $V$ is such that $\operatorname{dim} V_{p}<\infty$ for every $p$, then $\operatorname{Hom}_{A}(A \otimes V, A)=$ $A \otimes \operatorname{Hom}(V, K)=A \otimes V^{*}$. The following corollary follows from this equality and from (2.1) and (2.2).

(2.4) Corollary. Assume $A$ is augmented and either $M^{p}=N^{p}=0$ whenever $p<0$ or there is an integer $n \geq 0$ such that $A^{p}=0$ if $p>n$ and $\left(K \otimes_{A} M\right)_{r}=\left(K \otimes_{A} N\right)_{r}=0$ whenever $r<0$. Also assume $M$ and $N$ are $F^{2}$-free over $A$ and $\gamma: M \rightarrow N$ is a DG-module weak equivalence over $A$. Then the induced map $\operatorname{Hom}_{A}(\gamma, \mathrm{id}): \operatorname{Hom}_{A}(N, A) \rightarrow \operatorname{Hom}_{A}(M, A)$ is a $D G$-module weak equivalence over $A$.

Change of base. Let $A$ and $A^{\prime}$ be two DG-algebras, $M$ a DG-module over $A$ and $f: A \rightarrow A^{\prime}$ a DG-algebra map. Recall that the tensor product $A^{\prime} \otimes_{A} M$ is a DG-module over $A^{\prime}$ (with the differential $d_{A^{\prime}} \otimes_{A}$ id + id $\left.\otimes_{A} d_{M}\right)$ and is called the $D G$-module obtained from $M$ by the change of base $f$. If $M$ is $F^{2}$-free over $A$ then $A^{\prime} \otimes_{A} M$ in $F^{2}$-free over $A^{\prime}$ and, furthermore, if $f$ is a weak equivalence, then the natural projection $M \rightarrow A^{\prime} \otimes_{A} M$ 
is a weak equivalence, provided that either $M^{p}=0$ if $p<0$ or there is an integer $n \geq 0$ such that $A^{p}=0$ for $p>n$ and $\left(K \otimes_{A} M\right)_{r}=0$ whenever $r<0$. Under the same conditions and if $N$ is a DG-module over $A$ satisfying the same properties as $M$, if $\alpha: M \rightarrow N$ is a weak equivalence, then id $\otimes_{A} \alpha: A^{\prime} \otimes_{A} M \rightarrow A^{\prime} \otimes_{A} N$ is a weak equivalence.

3. Duality. In this paragraph we shall see that the functor $E \leadsto$ $\operatorname{Hom}_{A}(E, A)$ establishes, under certain conditions, a duality between the category of DG-algebras over $A$ which are free $A$-modules and the category of DG-coalgebras over $A$ which also are free $A$-modules.

(3.1) Proposition. Let $A$ be an augmented DG-algebra. The functor $\operatorname{Hom}_{A}(, A)$ establishes an equivalence between the DG-algebras $E$ over $A$ such that $E$ is a free $A$-module and $\operatorname{dim}\left(K \otimes_{A} E\right)^{p}<\infty$ for every $p$, and the DG-coalgebras $\mathcal{X}$ over $A$ such that $\mathfrak{X}$ is a free $A$-module and $\operatorname{dim}\left(K \otimes_{A} \mathcal{X}\right)_{p}<\infty$ for every $p$. This equivalence is functorial relatively to the change of base and preserves the $F^{2}$-property. Furthermore, there is a one-one correspondence between the DG-algebra maps $\sigma: E \rightarrow A$ such that $u \sigma=\mathrm{id}$, where $u: A \rightarrow E$ is the unity, and the augmentations of $\operatorname{Hom}_{A}(E, A)$.

Proof. Let $E$ be a DG-algebra over $A$, free as an $A$-module and such that $\operatorname{dim}\left(K \otimes_{A} E\right)^{p}<\infty$ for every $p$. Let

$$
\gamma: \operatorname{Hom}_{A}(E, A) \otimes_{A} \operatorname{Hom}_{A}(E, A) \rightarrow \operatorname{Hom}_{A}(E \otimes E, A)
$$

be the DG-algebra isomorphism over $A$ defined by

$$
\gamma\left(\varphi \otimes_{A} \psi\right)(x \otimes y)=(-1)^{\operatorname{deg} \psi \operatorname{deg} x} \varphi(x) \psi(y) .
$$

One can easily verify that, if $E$ is identified to $A \otimes\left(K \otimes_{K} E\right)$ as an $A$-module, then $\gamma$ is just the canonical isomorphism

$\left(A \otimes\left(K \otimes_{A} E\right)^{*}\right) \otimes_{A}\left(A \otimes\left(K \otimes_{A} E\right)^{*}\right)=A \otimes\left(K \otimes_{A} E\right)^{*} \otimes\left(K \otimes_{A} E\right)^{*}$.

The structure on $\operatorname{Hom}_{A}(E, A)$ of DG-coalgebras over $A$ is given by

$$
\Delta: \operatorname{Hom}_{A}(E, A) \rightarrow \operatorname{Hom}_{A}(E, A) \otimes_{A} \operatorname{Hom}_{A}(E, A)
$$

and

$$
\varepsilon: \operatorname{Hom}_{A}(E, A) \rightarrow A
$$

defined by $\Delta \varphi=\gamma^{-1} \circ\left(\Delta_{E} \circ \varphi\right)$ and $\varepsilon(\varphi)=\varphi(1)$.

One can directly verify that $\operatorname{Hom}_{A}(, A)$ is a functor, and that if $E$ is a $F^{2}$-free algebra over $A$, then $\operatorname{Hom}_{A}(E, A)$ is a $F^{2}$-free coalgebra over $A$. 
Also, if $A \rightarrow A^{\prime}$ is a DG-algebra map, then

$$
\operatorname{Hom}_{A^{\prime}}\left(A^{\prime} \otimes_{A} E, A^{\prime}\right) \cong A^{\prime} \otimes_{A} \operatorname{Hom}_{A}(E, A) .
$$

Let $\mathcal{X}$ be a DG-coalgebra over $A$, free as an $A$-module and such that $\operatorname{dim}\left(K \otimes_{A} \mathcal{X}\right)_{p}<\infty$ for every $p$. Then the maps

$$
\begin{aligned}
m: \operatorname{Hom}_{A}(\mathfrak{X}, A) \otimes_{A} \operatorname{Hom}_{A}(\mathfrak{X}, A) \\
\quad=\operatorname{Hom}_{A}(\mathfrak{X} \otimes \mathfrak{X}, A) \stackrel{\operatorname{Hom}_{A}(\Delta, \mathrm{id})}{\rightarrow} \operatorname{Hom}_{A}(\mathfrak{X}, A)
\end{aligned}
$$

and

$$
u: A \stackrel{\operatorname{Hom}_{A}(\varepsilon, \text { id })}{\rightarrow} \operatorname{Hom}_{A}(\mathcal{X}, A)
$$

define on $\operatorname{Hom}_{A}(\mathfrak{X}, A)$ a DG-algebra structure over $A$.

If $\mathcal{X}$ is a $F^{2}$-free DG-coalgebra over $A$, then $\operatorname{Hom}_{A}(\mathfrak{X}, A)$ is a $F^{2}$-free DG-algebra over $A$ and if $A \rightarrow A^{\prime}$ is a DG-algebra map, then

$$
\operatorname{Hom}_{A^{\prime}}\left(A^{\prime} \otimes \otimes_{A} \mathfrak{X}, A^{\prime}\right) \cong A^{\prime} \otimes \operatorname{Hom}_{A}(\mathfrak{X}, A) .
$$

One also verifies directly that the maps

$$
v: E \rightarrow \operatorname{Hom}_{A}\left(\operatorname{Hom}_{A}(E, A), A\right)
$$

and

$$
w: \mathfrak{X} \rightarrow \operatorname{Hom}_{A}\left(\operatorname{Hom}_{A}(\mathfrak{X}, A), A\right)
$$

defined by

$$
v(x)(\varphi)=(-1)^{\operatorname{deg} x \operatorname{deg} \varphi} \varphi(x), \quad w(\xi)(\varphi)=(-1)^{\operatorname{deg} \xi \operatorname{deg} \varphi} \varphi(\xi)
$$

are, respectively, a DG-algebra isomorphism over $A$ and a DG-coalgebra isomorphism over $A$.

Finally, if $\sigma: E \rightarrow A$ is a DG-algebra map such that $\sigma u=$ id, then $\eta$ : $A \rightarrow \operatorname{Hom}_{A}(E, A)$, defined by $\eta(1)=\sigma$, is an augmentation of $\operatorname{Hom}_{A}(E, A)$. Conversely if $\eta: A \rightarrow \operatorname{Hom}_{A}(E, A)$ is an augmentation, then $\eta(1): E \rightarrow A$ is a DG-algebra map such that $\eta(1) u=$ id.

The following proposition follows immediately from (2.4).

(3.2) Proposition. Let $A$ be an augmented DG-algebra. Assume there exists an integer $n \geq 0$ such that $A^{p}=0$ for $p>n$. Let $E$ and $E^{\prime}$ be two $F^{2}$-free algebras over $A$ such that $K \otimes_{A} E$ and $K \otimes_{A} E^{\prime}$ are finite dimensional in each degree. Then $\alpha: E \rightarrow E^{\prime}$ is a $D G$-algebra weak equivalence over $A$ if and only if $\operatorname{Hom}_{A}(\alpha, \mathrm{id}): \operatorname{Hom}_{A}\left(E^{\prime} A\right) \rightarrow \operatorname{Hom}_{A}(E, A)$ is a $D G$-coalgebra weak equivalence over $A$. 
Analogy with the Differential Forms Defined on the Base With Values in the CurRents on the Fiber.

Let $\Omega_{X}$ be the DG-algebra of differential forms on a differentiable manifold $X$. Let $p: X \rightarrow Y$ be a differentiable locally trivial fibre bundle and assume that $X$ and $Y$ are compact (this assumption is not essential).

Let $p^{*}: \Omega_{Y} \rightarrow \Omega_{X}$ be the DG-algebra map induced by $p$ and consider $\Omega_{X}$ with the structure of $\Omega_{Y}$-module induced by $p^{*}$. In this situation we can give the following interpretation for the functor $\operatorname{Hom}_{\Omega_{Y}}\left(, \Omega_{Y}\right)$ : it is the functor which associates to the bundle $p$ the "topological" 1 DG-coalgebra $\operatorname{Hom}_{\Omega_{Y}}^{\text {cont }}\left(\Omega_{X}, \Omega_{Y}\right)$ whose elements are the continuous $\Omega_{Y}$-linear maps of $\Omega_{X}$ in $\Omega_{Y}$. Such a map can be identified to a differential form on $Y$ with values in the vector bundle of currents on the fibers. This can be seen by noting that the elements of $\operatorname{Hom}_{\Omega_{Y}}^{\text {cont }}\left(\Omega_{X}, \Omega_{Y}\right)$ are the cross-sections of a sheaf on $Y$ and by considering the situation over an open $U \subset Y$ where the bundle is trivial.

If the fibers are oriented, an example of such a form of degree equal to the dimension of the fibre is given by the integration on the fibres.

\section{Quillen's functors $\varrho_{A}$ and $\bigodot_{A}$.}

The functor $\bigodot_{A}$. Let $A$ be a DG-algebra and $L$ a DG-Lie algebra over $A$. Let $S_{A}(s L)$ be the symmetric coalgebra over $A$ on the suspension of $L$ $\left((s L)_{p}=L_{p-1}\right)$. We shall denote by $C_{A}(L)$ the coalgebra $S_{A}(s L)$ over $A$ equipped with the differential defined by

$$
\begin{aligned}
d\left(s x_{1} \cdot s x_{2} \cdots s x_{n}\right)= & \sum_{k} \pm s x_{1} \cdots s d x_{k} \cdots s x_{n} \\
& +\sum_{i<j} \pm s\left[x_{i}, x_{j}\right] s x_{1} \cdots s \hat{x}_{i} \cdots s \hat{x}_{j} \cdots s x_{n}
\end{aligned}
$$

where $x_{1}, \ldots, x_{n}$ belong to $L$ (for the signs cf. [6] page 506).

Using the natural generalizations to DG-coalgebras over $A$ of Proposition 4.1 and Corollary 4.4 in Appendix B of [11] (pages 285-286) (cf. also [10] pages 441-444), one easily checks that $\bigodot_{A}$ is a functor and that the differential is completely determined by $d(s x)=-s d x$ and

$$
d\left(s x_{1} s x_{2}\right)=-(-1)^{\operatorname{deg} x_{1}} s\left[x_{1}, x_{2}\right], \quad \text { for } x, x_{1}, x_{2} \in L .
$$

\footnotetext{
${ }^{1}$ The tensor product is replaced by a well-adapted completion.
} 
Change of base: let $A \rightarrow A^{\prime}$ be a DG-algebra map. Then $\bigodot_{A^{\prime}}\left(A^{\prime} \otimes_{A} L\right)$ $=A^{\prime} \otimes_{A} \bigodot_{A}(L)$.

Note that $\mathcal{C}_{A}(L)$ is the natural generalization of the Koszul complex of chains on a Lie algebra.

The functor $\mathfrak{L}_{A}$. Let $A$ be a DG-algebra and $\mathscr{X}$ an augmented DGcoalgebra over $A$. Let $\eta: A \rightarrow \mathfrak{X}$ and $\varepsilon: \mathfrak{X} \rightarrow A$ be, respectively, the augmentation and the counity of $\mathfrak{X}$. Set $\bar{X}=\operatorname{Ker} \varepsilon$. Then $\bar{\xi}=\xi-\eta \varepsilon(\xi)$ defines a projection $\mathfrak{X} \rightarrow \overline{\mathcal{X}}$. We shall denote by $\varrho_{A}(\mathfrak{X})$ the free Lie algebra $L_{A}\left(s^{-1 \bar{X}}\right)$ on $s^{-1 \bar{X}}$ over $A$ equipped with the differential ([11] page 290) obtained by extending to a derivation the map

$$
d: s^{-1} \overline{\mathfrak{X}} \rightarrow L_{A}\left(s^{-1 \bar{X}}\right)
$$

given by

$$
d\left(s^{-1} \bar{\xi}\right)=-s^{-1} \overline{d \xi}-\frac{1}{2} \sum_{i}(-1)^{\operatorname{deg} \xi_{i}^{\prime}}\left[s^{-1} \overline{\xi_{i}^{\prime}}, s^{-1} \overline{\xi_{l}^{\prime \prime}}\right]
$$

if $\Delta \xi=\Sigma_{i} \xi_{l}^{\prime} \otimes_{A} \xi_{l}^{\prime \prime}$. (If $M$ is an $A$-module, the free graded Lie-algebra $L_{A}(M)$ on $M$ over $A$ can be built as a quotient of the non-associative and non-commutative free algebra on $M$ over $A$, see [1], page 181, ex. 13.)

Using the universal property of the free Lie algebra over $A$, one checks that $\rho_{A}$ is a functor.

Change of base: let $A \rightarrow A^{\prime}$ be a DG-algebra map. Then $\mathcal{L}_{A^{\prime}}\left(A^{\prime} \otimes_{A} \mathfrak{X}\right)$ $=A^{\prime} \otimes_{A} \mathcal{L}_{A}(\mathfrak{X})$.

The adjunction maps ([11] page 293, [10] page 442).

Let $L$ be a DG-Lie algebra over $A$. Using the universal property one extends the projection $s^{-1} S_{A}(s L) \rightarrow L$ to a DG-Lie algebra map

$$
\alpha: \varrho_{A} e_{A}(L) \rightarrow L
$$

Let $\mathcal{X}$ be an augmented DG-coalgebra over $A$. Assume $\mathcal{X}$ is connected in the sense of Quillen [11] (page 282), i.e. for a given augmentation $\eta: A \rightarrow \mathfrak{X}$ we have $\mathfrak{X}=\cup_{r \geq 0} F_{r} \mathfrak{X}$, where $F_{r} \mathfrak{X}$ is inductively defined by

$$
\begin{aligned}
& F_{0} \mathfrak{X}=\eta(A), \\
& F_{r} \mathcal{X}=\left\{\xi \in \mathfrak{X} \mid \Delta \xi-\left(\xi \otimes_{A} \eta(1)+\eta(1) \otimes_{A} \xi\right) \in F_{r-1} \mathfrak{X} \otimes_{A} F_{r-1} \mathcal{X}\right\} .
\end{aligned}
$$

(If $\mathcal{X}$ is a free-module over $A$ and $\left(K \otimes_{A} \mathfrak{X}\right)_{p}=0$ for $p<0$, then $\mathcal{X}$ is connected in the sense of Quillen.) Then, again using the generalization of 
Proposition 4.1 in Appendix B of [11], one sees that the $A$-module map $\mathcal{X} \rightarrow \bar{X} \rightarrow s L_{A}\left(s^{-1} \bar{X}\right)$ lifts to a DG-coalgebra map over $A$ :

$$
\beta: \mathcal{X} \rightarrow \mathcal{C}_{A} \mathscr{L}_{A}(\mathfrak{X}) \text {. }
$$

(4.1) Proposition. Let $A$ be an augmented DG-algebra and assume there is an integer $n \geq 0$ such that $A^{p}=0$ if $p>n$.

(1) If $f: L \rightarrow L^{\prime}$ is a DG-Lie algebra weak equivalence over $A$ and if $L$ and $L^{\prime}$ are $F^{2}$-free and such that $\left(K \otimes_{A} L\right)_{p}=\left(K \otimes_{A} L^{\prime}\right)_{p}=0$ for $p \leq 0$, then $\bigodot_{A}(f): \bigodot_{A}(L) \rightarrow \bigodot_{A}\left(L^{\prime}\right)$ is a $D G$-coalgebra weak equivalence over $A$.

(2) If $g: \mathfrak{X} \rightarrow \mathfrak{X}^{\prime}$ is a DG-coalgebra weak equivalence over $A$, if $\mathfrak{X}$ and $\mathfrak{X}^{\prime}$ are augmented, $F^{2}$-free and such that $\left(K \otimes_{A} \mathfrak{X}\right)_{p}=\left(K \otimes_{A} \mathfrak{X}^{\prime}\right)_{p}=0$ for $p<0$ and $\left(K \otimes_{A} \mathfrak{X}\right)_{0}=\left(K \otimes_{A} \mathcal{X}^{\prime}\right)_{0}=K$, then $\mathcal{L}_{A}(g): \mathcal{L}_{A}(\mathcal{X}) \rightarrow \mathcal{L}_{A}\left(\mathfrak{X}^{\prime}\right)$ is a DG-Lie algebra weak equivalence over $A$.

(3) If $L$ is a $F^{2}$-free DG-Lie algebra over $A$ such that $\left(K \otimes_{A} L\right)_{p}=0$ for $p \leq 0$ and $\mathcal{X}$ is an augmented $F^{2}$-free $D G$-coalgebra over $A$ such that $\left(K \otimes_{A} \mathfrak{X}\right)_{p}=0$ for $p<0$ and $\left(K \otimes_{A} \mathscr{X}\right)_{0}=K$, then $\alpha$ and $\beta$ are weak equivalences over $A$.

Proof. Note that if $L$ is a $F^{2}$-free DG-Lie algebra over $A$, then $\bigodot_{A}(L)$ is a $F^{2}$-free DG-coalgebra over $A$ and, also, if $\mathcal{X}$ is a $F^{2}$-free DG-coalgebra over $A$, then $\varrho_{A}(\mathcal{X})$ is a $F^{2}$-free DG-Lie algebra over $A$.

The proposition being true for $A=K([\mathbf{1 0}, 11])$, the general case follows from Corollary (2.2). For instance, to prove (1) consider the DG-Lie algebra map $f^{K}: L^{K} \rightarrow L^{\prime K}$ induced by $f$. By (2.2), $f^{K}$ is a weak-equivalence, hence $\mathcal{C}\left(f^{K}\right): \mathcal{C}\left(L^{K}\right) \rightarrow \mathcal{C}\left(L^{\prime K}\right)$ is also a weak equivalence. Thus, by (2.2), $e_{A}(f)$ is a weak equivalence over $A$. The same argument proves (2) and (3).

(4.2) Remark. The equivalence between (3) and (4) in Theorem 1, §1, follows from (4.1). This ends the proof of Theorem 1.

(4.3) Remark. Let $A$ be a DG-algebra and $L$ a DG-Lie algebra over $A$. By restriction of scalars, consider $L$ as a DG-Lie algebra over $K$. Let $S_{u}: S(s L) \rightarrow S_{A}(s L)$ be the natural coalgebra map over the unity $u: K \rightarrow A$ (see [6], page 521). One can easily check that $S_{u}: \mathcal{C}(L) \rightarrow \bigodot_{A}(L)$ is a DG-coalgebra map over $u$. We shall need this map in the next paragraph.

The $D G$-algebra $\bigodot_{A}^{*}(L)$. Let $A$ be an augmented DG-algebra and $L$ a DG-Lie algebra over $A$ and set $\bigodot_{A}^{*}(L)=\operatorname{Hom}_{A}\left(\bigodot_{A}(L), A\right)$. If $L$ is free as an $A$-module and $\operatorname{dim}\left(K \otimes_{A} L\right)_{p}<\infty$ for every $p$, then $\bigodot_{A}^{*}(L)$ is naturally 
equipped with a structure of DG-algebra over $A$, free as an $A$-module. We shall call it the DG-algebra of cochains on $L$ over $A$.

Assume $\left(K \otimes_{A} L\right)_{p}=0$ for $p<0$ and set $V=s\left(K \otimes_{A} L\right)^{*}$. Then, as an algebra, $\bigodot_{A}^{*}(L)$ is the free algebra $A(V)$ over $A$ generated by $V$.

(4.4) Proposition. Let $E$ be a DG-algebra over A, free as an A-module and such that $\operatorname{dim}\left(K \otimes_{A} E\right)^{p}<\infty$ for every $p$. Then $E$ is the $D G$-algebra of cochains on a DG-Lie algebra over $A$ if and only if there exists a (strictly) positively graded vector space $V$, finite dimensional in each degree, such that $E$ is the free algebra $A(V)$ on $V$ over $A$ and the differential $d_{E} v$ of an element $v \in V$ (as element of $E$ ) is exactly the sum of a linear term $d_{1} v$ and $a$ quadratic term $d_{2} v$ (i.e. $d_{1} v$ is a sum of terms of the form $a \otimes w, a \in A$, $w \in V$, and $d_{2} v$ is a sum of terms of the form $a \otimes v_{1} \cdot v_{2}, a \in A, v_{1}, v_{2} \in$ $V)$.

Proof. Assume $E=e_{A}^{*}(L)$ for some DG-Lie algebra $L$ such that $\left(K \otimes_{A} L\right)_{p}=0$ for $p<0$. As above, set $V=s\left(K \otimes_{A} L\right)^{*}$.

Let $\left\{l_{i}\right\}$ be a base of $K \otimes_{A} L, l_{i}^{*}$ the dual base of $\left(K \otimes_{A} L\right)^{*}$ and set $v_{\imath}=s l_{i}^{*}$. Let

$$
d\left(1 \otimes l_{i}\right)=\sum_{j} a_{i}^{j} \otimes l_{j}
$$

and

$$
\left[1 \otimes l_{i}, 1 \otimes l_{j}\right]=\sum_{k} c_{i j}^{k} \otimes l_{k} .
$$

The differential of $\mathcal{C}_{A}(L)$ is completely determined by

$$
d\left(s\left(1 \otimes l_{i}\right)\right)=-s\left(\sum_{j} a_{i}^{j} \otimes l_{J}\right)
$$

and

$$
\begin{aligned}
d\left(s\left(1 \otimes l_{i}\right) \cdot s\left(1 \otimes l_{j}\right)\right) & =-(-1)^{\operatorname{deg} l_{i}} s\left[1 \otimes l_{i}, 1 \otimes l_{j}\right] \\
& =-(-1)^{\operatorname{deg} l_{l}} s\left(\sum_{k} c_{i j}^{k} \otimes l_{k}\right) .
\end{aligned}
$$

It follows that $d v_{h}$ is completely determined by

$$
\left(d v_{h}\right)\left(s\left(1 \otimes l_{i}\right)\right)= \pm a_{i}^{h}
$$

and

$$
\left(d v_{h}\right)\left(s\left(1 \otimes l_{i}\right) \cdot s\left(1 \otimes l_{j}\right)\right)= \pm c_{i j}^{h}
$$


The signs are + or - if, respectively, $\operatorname{deg} v_{h}+\operatorname{deg} a_{i}^{h}\left(1+\operatorname{deg} v_{h}\right)$ and $\operatorname{deg} v_{h}+\operatorname{deg} l_{i}+\operatorname{deg} c_{i j}^{h}\left(1+\operatorname{deg} v_{h}\right)$ are even or odd.

Hence

$$
d v_{h}=\sum_{i} \pm a_{i}^{h} \otimes v_{i}+\sum_{i, j} \pm \frac{1}{2} c_{i j}^{h} v_{i} \cdot v_{j},
$$

for $\left(v_{i} \cdot v_{j}\right)\left(s\left(1 \otimes l_{i}\right) \cdot s\left(1 \otimes l_{j}\right)\right)=2$.

We have to verify now that if $E=A(V)$ and the restriction to $V$ of the differential $d_{E}$ is the sum $d_{1}+d_{2}$ of a linear term and a quadratic term, then $E=\bigodot_{A}^{*}(L)$ for some DG-Lie algebra $L$ over $A$.

Let $D_{1}$ and $D_{2}$ be the derivations on $E$ defined by extending $D_{1}(a)=$ $d_{A} a, D_{1}(v)=d_{1} v$ and $D_{2}(a)=0, D_{2}(v)=d_{2} v$, for $a \in A$ and $v \in V$. Then we have $d_{E}=D_{1}+D_{2}$ and

$$
d_{E}^{2}=0=D_{1}^{2}+D_{1} D_{2}+D_{2} D_{1}+D_{2}^{2}=0,
$$

hence

$$
D_{1}^{2}=D_{2}^{2}=0 \text { and } D_{1} D_{2}+D_{2} D_{1}=0
$$

Set

$L^{K}=\left(s^{-1} V\right)^{*}=\operatorname{Hom}\left(s^{-1} V, K\right)$ and $L=A \otimes L^{K}=\operatorname{Hom}\left(s^{-1} V, A\right)$.

We shall define on $L$ a structure of a DG-Lie algebra over $A$ such that $\mathcal{C}_{A}^{*}(L)=E$.

First fix the following notation. For an $A$-module $M$, denote by $T_{A}(M)$ the tensor algebra on $M$ over $A$. As an $A$-module $T_{A}(M)$ equals $\bigoplus_{n \geq 0}\left(\otimes_{A}^{n} M\right)$, where $\otimes_{A}^{0} M=A$ and $\otimes_{A}^{n} M=M \otimes_{A} M \otimes_{A} \cdots \otimes_{A} M(n$ times).

If $U$ is a graded vector space, we shall denote by

$$
\lambda: A(U) \rightarrow T_{A}(A \otimes U)
$$

the algebra injective map over $A$ given, for $a \in A$ and $u_{1}, u_{2}, \ldots, u_{p} \in U$, by

$$
\begin{aligned}
& \lambda\left(a \cdot u_{1} u_{2} \cdots u_{p}\right) \\
& \quad=\frac{1}{n !} \sum_{\sigma \in \Sigma_{p}} \pm a\left(1 \otimes u_{\sigma(1)}\right) \otimes_{A}\left(1 \otimes u_{\sigma(2)}\right) \otimes_{A} \cdots \otimes_{A}\left(1 \otimes u_{\sigma(p)}\right),
\end{aligned}
$$

where $\Sigma_{p}$ is the group of permutations of $p$ objects and where the sign is defined by $\pm u_{\sigma(1)} \cdot u_{\sigma(2)} \cdots u_{\sigma(p)}=u_{1} u_{2} \cdots u_{p}$ in $K(U)$. 
With this notation, we have, for $a \in A$ and $v \in V, \lambda D_{2}(a v) \in(A \otimes$ $V) \otimes_{A}(A \otimes V)$, hence $D_{2}^{\prime}=\lambda D_{2}$ is a map

$$
D_{2}^{\prime}: A \otimes V \rightarrow(A \otimes V) \otimes_{A}(A \otimes V)=A \otimes V \otimes V .
$$

Let

$$
\begin{aligned}
& \chi: \operatorname{Hom}(V, A) \otimes_{A} \operatorname{Hom}(V, A) \\
& \quad=\operatorname{Hom}_{A}(A \otimes V \otimes V, A) \rightarrow \operatorname{Hom}_{A}(A \otimes V, A)=\operatorname{Hom}(V, A)
\end{aligned}
$$

be the dual map and let $\omega: \operatorname{Hom}\left(s^{-1} V, A\right) \rightarrow \operatorname{Hom}(V, A)$ be the isomorphism given by $\omega(\xi)(v)=\xi\left(s^{-1} v\right)$. Then

$$
[,]: L \otimes_{A} L \rightarrow L,
$$

defined by $[]=,\omega^{-1} \circ \chi \circ\left(\omega \otimes_{A} \omega\right)$, is a bracket, i.e. for every $x_{1}, x_{2}$, $x_{3} \in L$, we have

(i)

$$
\left[x_{1}, x_{2}\right]=-(-1)^{\operatorname{deg} x_{1} \operatorname{deg} x_{2}}\left[x_{2}, x_{1}\right]
$$

and

(ii)

$$
\left[x_{1},\left[x_{2}, x_{3}\right]\right]=\left[\left[x_{1}, x_{2}\right], x_{3}\right]+(-1)^{\operatorname{deg} x_{1} \operatorname{deg} x_{2}}\left[x_{2},\left[x_{1}, x_{3}\right]\right] .
$$

Indeed, these formulas are equivalent to

(i)'

$$
\chi\left(\xi_{1}, \xi_{2}\right)=(-1)^{\operatorname{deg} \xi_{1} \xi_{2}} \chi\left(\xi_{2}, \xi_{1}\right)
$$

and

(ii)'

$$
\begin{aligned}
\chi\left(\xi_{1} \otimes_{A} \chi\left(\xi_{2} \otimes_{A} \xi_{3}\right)\right)= & \chi\left(\chi\left(\xi_{1} \otimes_{A} \xi_{2}\right) \otimes_{A} \xi_{3}\right) \\
& +(-1)^{\operatorname{deg} \xi_{1} \operatorname{deg} \xi_{2}} \chi\left(\xi_{2} \otimes_{A} \chi\left(\xi_{1} \otimes_{A} \xi_{3}\right)\right),
\end{aligned}
$$

for $\xi_{1}, \xi_{2}$ and $\xi_{3} \in \operatorname{Hom}(V, A)$. Now, (i)' follows from the definition of $\chi$ and, by duality, (ii)' is true if and only if the following formula is true:

(ii)"

$$
\left(D_{2}^{\prime} \otimes_{A} \mathrm{id}\right) D_{2}^{\prime}=\left(\left(\mathrm{id} \otimes_{A} D_{2}^{\prime}\right)+\left(\mathrm{id} \otimes_{A} T\right)\left(D_{2}^{\prime} \otimes_{A} \mathrm{id}\right)\right) D_{2}^{\prime} .
$$

But

$$
0=\lambda\left(D_{2}^{2}\right)=\left(D_{2}^{\prime} \otimes_{A} \mathrm{id}\right) D_{2}^{\prime}-\left(\left(\mathrm{id} \otimes_{A} E_{2}^{\prime}\right)+\left(\mathrm{id} \otimes_{A} T\right)\left(D_{\underline{E}}^{\prime} \otimes_{A} \mathrm{id}\right)\right) D_{2}^{\prime},
$$

as one can check directly. $\left(T\right.$ is defined by $T\left(x \otimes_{A} y\right)=(-1)^{\operatorname{deg} x \operatorname{deg} y} y$ $\otimes_{A} x$.) 
Now set $d=\omega^{-1} \circ \operatorname{Hom}_{A}\left(D_{1}\right.$, id $) \circ \omega: L \rightarrow L$. This defines a differential on $L$ : the fact that $d^{2}=0$ follows directly from $D_{1}^{2}=0$, and the fact that $d[]=,[],\left(d \otimes_{A}\right.$ id $\left.+\mathrm{id} \otimes_{A} d\right)$ follows from $\lambda\left(D_{1} D_{2}+D_{2} D_{1}\right)$ $=0$.

As an algebra we have $\mathcal{C}_{A}^{*}(L)=A(V)$ and to see that actually $\bigodot_{A}^{*}(L)=E$, note that the differentials of an element $v \in V$ are equal. This completes the proof of the proposition.

(4.5) Consequence. Let $A$ be a finite dimensional DG-algebra and $L$ be a DG-Lie-algebra over $A$ such that $\left(K \otimes_{A} L\right)_{p}=0$ for $p \leq 0$ and $\operatorname{dim}\left(K \otimes_{A} L\right)_{p}<\infty$ for every $p$ and free as an $A$-module. If $A$ is a model for a space $Y$, then $L$ is a model for a fibration $p: X \rightarrow Y$ with a given cross-section $s: Y \rightarrow X$. Let $s^{\prime}: Y \rightarrow X$ be another cross-section of $p$. Then there exists a differential $d^{\prime}$ on $L$ such that if $L^{\prime}$ denotes $L$ equipped with $d^{\prime}$, then $L^{\prime}$ is a model for the fibration $p$ equipped with $s^{\prime}$.

Proof. The DG-algebra $\bigodot_{A}^{*}(L)$ over $A$ equipped with the natural augmentation $\eta$ : $\mathcal{C}_{A}^{*}(L) \rightarrow A$ is a model for the fibration $p$ equipped with the cross-section $s$. Let $\sigma: \mathcal{C}_{A}^{*}(L) \rightarrow A$ be the augmentation corresponding to $s^{\prime}$. Let $\mathcal{C}_{A}^{*}(L)=A(V)$ and define an algebra isomorphism $\varphi: A(V) \rightarrow$ $A(V)$ over $A$ by extending $\varphi(1 \otimes v)=1 \otimes v-\sigma(1 \otimes v) \otimes 1$ into an $A$-algebra map. Then the following diagram commutes:

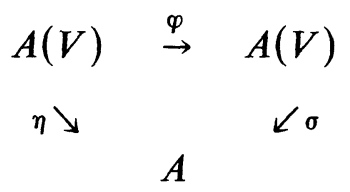

Set $\tilde{d}(1 \otimes v)=\varphi^{-1} d \varphi(1 \otimes v)$. This defines a differential on $A(V)$ such that $(d-\tilde{d})(1 \otimes v)$ is a linear term of the form $\Sigma_{i} a_{i} v_{i}, a_{i} \in A$, $v_{i} \in V$, and if $E$ equals $A(V)$ equipped with $\tilde{d}$, then $\varphi: E \rightarrow \mathcal{C}_{A}^{*}(L)$ is a DG-algebra map over $A$.

Now, it follows from Proposition (4.4) that there exists a DG-Lie algebra $L^{\prime}$ over $A$ such that $E=\mathcal{C}_{A}^{*}\left(L^{\prime}\right)$ and as the differential on $E$ differs from that on $\mathcal{C}_{A}^{*}(L)$ by a linear term, as a Lie algebra, $L^{\prime}$ equals $L$.

We shall illustrate this consequence in the examples of $\S 6$.

5. The model $L_{+}$for the space of cross-sections.

Definition. Let $A$ be a DG-algebra. We say that a DG-algebra $E$ over $A$ is free nilpotent over $A$ if $E$ is a free algebra $A(V)$ (see Definitions and conventions in $\S 1$ ) where $V$ is a graded vector space such that $V^{p}=0$ 
for $p \leq 0, \operatorname{dim} V^{p}<\infty$ for every $p$, and such that there exists an increasing filtration $V(1) \subset V(2) \subset V(3) \subset \cdots \subset V(k) \subset \cdots$ of $V$ for which $d(A(V(k))) \subset A(V(k-1))$.

(5.1) Sullivan's model. Let $A$ be a finite dimensional DG-algebra such that $A^{0}=K$ and let $C=A^{*}$ be the dual DG-coalgebra. Let $E$ be a free nilpotent DG-algebra over $A$ and let $\sigma: E \rightarrow A$ be an augmentation of $E$. Conditional to a change of base in the graded vector space of generators (and the corresponding change in the differential) one can assume $\sigma$ : id $\otimes \varepsilon$, where $\varepsilon: Q(V) \rightarrow K$ is the natural augmentation (as above, $V$ is the space of generators of $E$ over $A$ ) (see [8]). Below we shall systematically make this assumption.

Sullivan has suggested [12] (page 314) the following model for the space of cross-sections which are in the homotopy class of the section corresponding to $\sigma$. Consider the free algebra $\mathbf{Q}(V \otimes C)$ (which in general is not positively graded) and the algebra map

$$
e^{\prime}: A(V) \rightarrow A \otimes \mathbf{Q}(V \otimes C)=\operatorname{Hom}(C,(V \otimes C))
$$

obtained by setting $e^{\prime}(1 \otimes v)(c)=v \otimes c$ and extending as an $A$-algebra map. Then the derivation on $\mathbf{Q}(V \otimes C)$ given by

$$
d(v \otimes c)=e(d v)(c)+(-1)^{\operatorname{deg} v} v \otimes d c
$$

is the unique differential on $\mathbf{Q}(V \otimes C)$ such that $e^{\prime}$ is a DG-algebra map over $A$. With this differential, the couple $\left(e^{\prime},(V \otimes C)\right)$ satisfies the following universal property: for every DG-algebra map $\Phi: E \rightarrow A \otimes B$ over $A$, where $B$ is any DG-algebra, there exists a DG-algebra map $\varphi$ : $\mathbf{Q}(V \otimes C) \rightarrow B$ such that the following diagram commutes:

$$
\begin{array}{ccc}
E & \stackrel{e^{\prime}}{\rightarrow} & A \otimes \mathbf{Q}(V \otimes C) \\
& & \swarrow \text { id } \otimes \varphi
\end{array}
$$

In fact, $\varphi$ is determined by $\varphi(v \otimes c)=\Phi(1 \otimes v)(c)$.

Let $I$ be the ideal of $\mathbf{Q}(V \otimes C)$ generated by the elements of $V \otimes C$ of degree $\leq 0$ and their differentials and let $\Gamma$ be the quotient DG-algebra $\mathrm{Q}(V \otimes C) / I$. Set $e: E \rightarrow A \otimes \Gamma$ for the DG-algebra map over $A$ given by $e^{\prime}$ followed by the natural projection. The DG-algebra $\Gamma$ is positively 
graded and the couple $(e, \Gamma)$ verifies the universal property

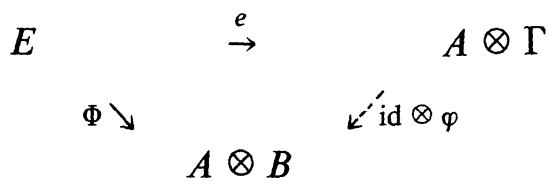

for any DG-algebra (positively graded) $B$ such that $B^{0}=K$ and for every map $\Phi$ of augmented DG-algebras over $A$.

(5.2) Explicit formulas for $e$. Let $U$ be the subspace of $V \otimes C$ of all elements of degree $\leq 0$ and their differentials (for the induced differential). Let $(V \otimes C)^{+}$be the quotient vector space $V \otimes C / U$. Then we have an algebra isomorphism $\Gamma \approx \mathbf{Q}\left((V \otimes C)^{+}\right)$.

Let $\left\{c_{i}\right\}$ be a base of $C$ (as a vector space) and $\left\{a_{i}\right\}$ be the dual base of $A$. Let $v_{i}$ be the class of $v \otimes c_{i}$ in $(V \otimes C)^{+}$for every $v \in V$. Then

$$
e(1 \otimes v)=\sum_{i} a_{i} \otimes v_{i}
$$

This is the evaluation as described in [12] and in [7]. This follows from the fact that the inverse of the canonical isomorphism $\Gamma \otimes A \stackrel{\approx}{\rightarrow} \operatorname{Hom}(C, \Gamma)$ (given by $(\xi \otimes a)(c)=\xi a(c))$ is defined by $\alpha \mapsto \Sigma_{i} \alpha_{i} \otimes a_{i}$ if $\alpha_{i}=\alpha\left(c_{i}\right)$.

Let. $W=V^{*}$ and $(A \otimes W)_{+}$be the sub-space of $A \otimes W$ dual to $(V \otimes C)^{+}$. Then we have an algebra isomorphism

$$
A \otimes \Gamma \approx \operatorname{Hom}\left(S\left((A \otimes W)_{+}\right), A\right),
$$

and if we identify $E$, as an $A$-algebra, to $A \otimes \operatorname{Hom}(S(W), K)$, then for every $\lambda \in \operatorname{Hom}(S(W), K), a_{1} \otimes w_{1}, a_{2} \otimes w_{2}, \ldots, a_{p} \otimes w_{p} \in(A \otimes W)_{+}$, we have

$$
\begin{array}{r}
e(1 \otimes \lambda)\left(a_{1} \otimes w_{1}, a_{2} \otimes w_{2}, \ldots, a_{p} \otimes w_{p}\right) \\
= \pm a_{1} a_{2} \cdots a_{p} \lambda\left(w_{1}, w_{2}, \ldots, w_{p}\right) .
\end{array}
$$

\section{The Functorial Property of Sullivan's Model.}

(5.3) Proposition. Let $E$ and $E^{\prime}$ be augmented free nilpotent $D G$-algebras over $A$. Assume there exists a weak equivalence $\psi: E \rightarrow E^{\prime}$ over $A$. Then $\Psi$ induces a DG-algebra weak equivalence $\Gamma(\psi): \Gamma(E) \rightarrow \Gamma\left(E^{\prime}\right)$.

Proof. As an algebra we have $E=A(V)$. Let $d^{\prime}$ be the differential on $\mathbf{Q}(V)=K \otimes_{A} E$ induced by the differential $d$ of $E$. For $v \in V$ we have $d^{\prime} v=w+\left(\right.$ sum of elements of the form $v_{1} \cdot v_{2} \cdots v_{n}, n>1, v_{l} \in V$ ) 
where $w \in V$. Set $d_{V} v=w$. This defines a differential on $V$ for $0=$ $d^{\prime}\left(d^{\prime} v\right)=d^{\prime} w+\left(\right.$ sum of elements of the form $v_{1}^{\prime} \cdot v_{2}^{\prime} \cdots v_{n}^{\prime}, n>1, v_{i}^{\prime} \in$ $V)$, and $d^{\prime} w=w^{\prime}+(\operatorname{sum} \cdots)$, and it follows that $w^{\prime}=d_{V}^{2} v=0$. Let $H(V)$ be the homology of $V$ relatively to $d_{V}$, and let $c: H(V) \rightarrow V$ be a monomorphism given by choosing for each class $\nu \in H(V)$ a cycle $c(\nu)$. Set $H=c(H(V))$. Then $V$ can be decomposed into the direct sum of three subspaces $H \oplus B \oplus C$ such that $d_{V}$ is an isomorphism (of degree one) of $C$ onto $B$.

Set $U=B \oplus C$ with the differential $d_{V}$ and consider the algebra $A \otimes K(U)$ with the differential defined by $d(a \otimes u)=d a \otimes \mathrm{id}+\mathrm{id} \otimes$ $d_{V} u$. Let $\Phi: A \otimes K(U) \rightarrow E$ be the DG-algebra map over $A$ defined by $\Phi(1 \otimes y)=1 \otimes y$ if $y \in C, \Phi(1 \otimes x)=d(1 \otimes y)$ if $x \in B, x=d_{V} y$, and extending into an $A$-algebra map.

Set $A^{\prime}=A \otimes \mathbf{Q}(U)$ and consider $E$ with the structure of DG-module over $A^{\prime}$ induced by $\Phi$. Then the DG-algebra $\Re=K \otimes_{A^{\prime}} E$ over $A$ is, as an algebra, equal to $A(H)$ and is in fact Sullivan's free minimal nilpotent model of $E$ over $A$ [12] (page 289).

Let $j: E \rightarrow$ T be the natural projection. It is a weak equivalence.

To see this consider the spectral sequence associated to the natural filtration of $E$ and $\Re$ as $A$-modules. Then $j$ induces an isomorphism on the $E_{1}$ terms, for the DG-algebra map $j^{K}: K \otimes_{A} E \rightarrow K \otimes_{A}$ N induced by $j$ is a weak equivalence. To prove this last statement, note that $j^{K}$ is equal to the natural projection $j^{K}: \mathbf{Q}(V) \rightarrow K \otimes_{\mathbf{Q}(U)} \mathbf{Q}(V)=\mathbf{Q}(H)$, where $\mathbf{Q}(V)$ is equipped with the structure of $\mathbf{Q}(U)$-module induced by $\Phi^{K}: \mathbf{Q}(U) \rightarrow$ $\mathbf{Q}(V)$. Now, let $\mathbf{Q}(V)$ and $\mathbf{Q}(H)$ be filtered by the length: an element of $\mathbf{Q}(V)$ or $\mathbf{Q}(H)$ is said to be of filtration $p$ if it can be written as a finite sum of elements of the form $x_{1} \cdot x_{2} \cdots x_{q}$ with $q \geq p\left(x_{i} \in V, H\right)$. Then $j^{K}$ induces an isomorphism on the $E_{1}$ terms of the associated spectral sequences. Applying the mapping theorem, it follows that $j^{K}$ induces an isomorphism on homology.

Now, as $\Re$ is free minimal on $A$, there exists [12] (page 252) a DG-algebra map over $A, \rho: \Re \rightarrow E$, which is a weak equivalence and such that $j \circ \rho=$ identity. It follows that the DG-algebra map over $A$, $\Re \otimes \mathbf{Q}(U) \rightarrow E$, defined by $(\xi \otimes u) \rightarrow \rho(\xi) \Phi(1 \otimes u)$, is an isomorphism, for it induces an isomorphism on cohomology and its projection onto the generators is the identity.

Let $h: \mathbf{Q}(U) \rightarrow \mathbf{Q}(U)(t, d t)$ be the homotopy (in the sense of [3], page 251) defined by $h(u)=u \otimes t-(-1)^{\operatorname{deg} u} u^{\prime} \otimes d t$ if $u=d u^{\prime}+u^{\prime \prime}, u^{\prime}, u^{\prime \prime}$ $\in C$. Then $h_{t=1}=$ identity and $h_{t=0}=\varepsilon: S(V) \rightarrow K$.

Let $\bar{h}: E \rightarrow E(t, d t)$ be the homotopy defined by id $\otimes h$, after identifying $E$ to $\Re \otimes Q(V)$. Then $\bar{h}_{t=1}=\mathrm{id}_{E}$ and $\bar{h}_{t=0}=\mathrm{id} \otimes \varepsilon$. 
Let $\Gamma(E)$ and $\Gamma(\Re)$ be Sullivan's models as defined in (5.1) corresponding to $E$ and $\mathfrak{T}$. Then by the universal property there exists one and only one DG-algebra map $\tilde{h}: \Gamma(E) \rightarrow \Gamma(E)(t, d t)$ such that the following diagram commutes:

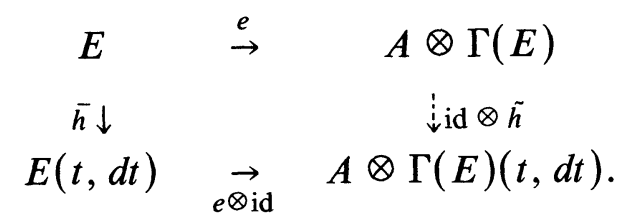

Using again the universal property, there exists a DG-algebra map $\Gamma(j): \Gamma(E) \rightarrow \Gamma(\Re)$ such that the following diagram commutes:

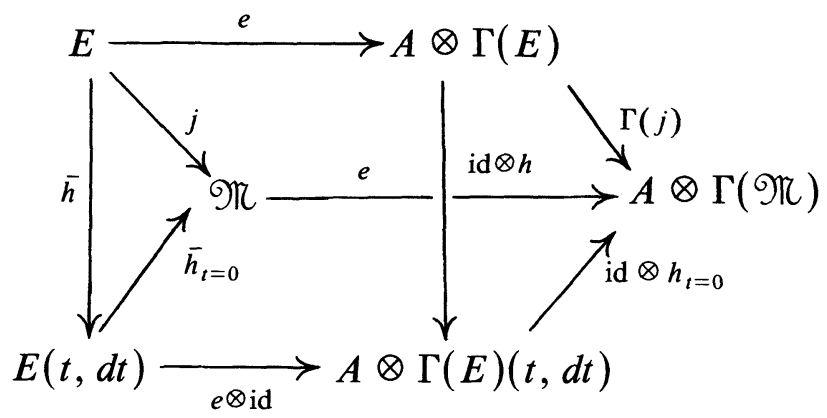

In particular $\Gamma(j)$ is a weak equivalence.

Now, in the same way as above one gets a DG-algebra weak equivalence $j^{\prime}: E^{\prime} \rightarrow \Re^{\prime}$ over $A$, and thus a weak equivalence $\Gamma\left(j^{\prime}\right): \Gamma\left(E^{\prime}\right) \rightarrow$ $\Gamma\left(\mathfrak{K}{ }^{\prime}\right)$, where $\mathfrak{K}^{\prime}$ is Sullivan's free minimal nilpotent model of $E^{\prime}$ over $A$.

Let $\psi: E \rightarrow E^{\prime}$ be a DG-algebra weak equivalence over $A$ and let $\alpha$ : $\mathfrak{K} \rightarrow \mathfrak{K}^{\prime}$ be the DG-algebra map defined by $\alpha=j^{\prime} \circ \psi \circ \rho: \mathfrak{N} \rightarrow E \rightarrow$ $E^{\prime} \rightarrow \Re^{\prime}$. It is a weak equivalence, hence an isomorphism. The map $\rho$ : $\Re \rightarrow E$ induces a DG-algebra map $\Gamma(\rho): \Gamma(\Re) \rightarrow \Gamma(E)$ such that $\Gamma(j) \circ \Gamma(\rho)=\Gamma(j \circ \rho)=$ id. In particular, $\Gamma(\rho)$ is a weak equivalence. Now, the induced isomorphism $\Gamma(\alpha): \Gamma(\mathfrak{R}) \rightarrow \Gamma\left(\Re^{\prime}\right)$ is equal to $\Gamma\left(j^{\prime}\right) \circ \Gamma(\psi) \circ \Gamma(\rho)$ and it follows that $\Gamma(\psi)$ is a weak equivalence.

(5.4) RemarK. Let $A$ be as above and let $E$ be a DG-algebra over $A$ such that $E=A(V)$ as an algebra over $A$, where $V$ is such that $V^{p}=0$ for $p \leq 1$ and $\operatorname{dim} V^{p}<\infty$ for every $p$. Assume, furthermore, that $E$ is a $F^{2}$-free module over $A$. Then $E$ is a free nilpotent DG-algebra over $A$. To prove this, proceed as in the beginning of the proof of (5.3) to show that $E$ is the tensor product of a free minimal nilpotent DG-algebra over $A$ and an acyclic DG-algebra. 
A Model in the Category of DG-Lie Algebras. (End of the proof of Theorem 2, §1.)

As above, let $A$ be a finite dimensional DG-algebra such that $A^{0}=K$ and let $C=A^{*}$ be the dual DG-coalgebra. Let $L$ be a $F^{2}$-free DG-Lie algebra over $A$ such that $\left(K \otimes_{A} L\right)_{p}=0$ for $p \leq 0$ and $\operatorname{dim}\left(K \otimes_{A} L\right)_{p}<\infty$ for every $p$. If $K=\mathbf{Q}$ and $A$ is a model for a space $Y$, then $L$ is a model for a fibration over $Y$ with a given cross-section (Theorem 1, §1).

Set $E=\bigodot_{A}^{*}(L)$ (cf. §4). It follows from Remark (5.4) that $E$ is a free nilpotent DG-algebra over $A$.

By restriction of scalars, consider $L$ as a DG-Lie algebra over $K$ and let $L_{+}$be the DG-Lie subalgebra of $L$ consisting of the elements of positive degrees and the cycles of degree zero. Set

$$
e^{*}\left(L_{+}\right)=\operatorname{Hom}\left(\bigodot\left(L_{+}\right), K\right) \text {. }
$$

We wish to show that $\Gamma(E)=\mathcal{C}^{*}\left(L_{+}\right)$.

Recall that we have algebra isomorphisms $\Gamma(E) \cong \mathbf{Q}\left((V \otimes C)^{+}\right) \cong$ $\operatorname{Hom}\left(S\left(A \otimes V^{*}\right)_{+}, K\right)$ (see (5.2)). We also have an algebra isomorphism $\mathcal{C}^{*}\left(L_{+}\right) \cong \operatorname{Hom}\left(S\left(s L_{+}\right) K\right)$, and to see that $s L_{+} \cong\left(A \otimes V^{*}\right)_{+}$ as a vector space, note that $\operatorname{Hom}\left(s L_{+}, K\right) \cong(V \otimes C)^{+}$, for the differential induced on $V \otimes C$ is precisely the dual to the differential on $s L$. It follows that we have an algebra isomorphism $\Gamma(E) \cong \mathrm{C}^{*}\left(L_{+}\right)$.

We must now show that the differential on $\bigodot^{*}\left(L_{+}\right)$is the unique differential such that the evaluation map $e: E \rightarrow A \otimes \bigodot^{*}\left(L_{+}\right)$is a DG-algebra map. Let

$$
\text { ev: } e\left(L_{+}\right) \rightarrow \bigodot_{A}(L)
$$

be the DG-coalgebra map over $u: K \rightarrow A$ defined by the natural injective map $\mathcal{C}\left(L_{+}\right) \rightarrow \mathcal{C}(L)$ followed by the map $S_{u}: \mathcal{C}(L) \rightarrow \mathcal{C}_{A}(L)$ (see Remark (4.3)). Let

$$
\text { ev: } \operatorname{Hom}_{A}\left(\bigodot_{A}(L), A\right) \rightarrow A \otimes e^{*}\left(L_{+}\right)
$$

be the restriction to $\operatorname{Hom}_{A}\left(\bigodot_{A}(L), A\right)$ of the natural map

$\operatorname{Hom}(\widetilde{\mathrm{ev}}, \mathrm{id}): \operatorname{Hom}\left(\bigodot_{A}(L), A\right) \rightarrow \operatorname{Hom}\left(\mathcal{C}\left(L_{+}\right), A\right)=A \otimes \mathcal{C}^{*}\left(L_{+}\right)$.

Then ev is a DG-algebra map over $A$ and, in fact, ev $=e$. To see this, one can directly check that if $a, a_{1}, a_{2}, \ldots, a_{p} \in A, \lambda \in \mathcal{C}^{*}\left(K \otimes_{A} L\right)$, $x_{1}, x_{2}, \ldots, x_{p} \in K \otimes_{A} L$, then (see (5.2)).

$$
\begin{array}{r}
\operatorname{ev}(a \otimes \lambda)\left(a_{1} \otimes s x_{1}, a_{2} \otimes s x_{2}, \ldots, a_{p} \otimes s x_{p}\right) \\
= \pm a a_{1} a_{2} \cdots a_{p} \lambda\left(s x_{1}, s x_{2}, \ldots, s x_{p}\right) .
\end{array}
$$


Assume $K=\mathbf{Q}$ and $A$ is a model for a space $Y$. Then $L$ is a model for a filtration $p: X \rightarrow Y$ with a given cross-section $s: Y \rightarrow X$ (Theorem 1, §1). Let $\Gamma_{s}$ be the space of cross-sections of $p$ homotopic to $s$. The proof of Theorem $2(\$ 1)$ now ends by using the theorem proved by Haefliger in [8] (Theorem 3.2), namely that if $E$ is a free nilpotent DG-algebra over $A$ which is a model for the fibration $p$, then $\Gamma(E)$ is a model for the space $\Gamma_{s}$.

\section{Examples.}

(6.1) The trivial fibration $Y \times Z$ and the constant map. Let $Y$ be a nilpotent space and $A$ a finite dimensional DG-algebra which is a model for $Y$. Let $Z$ be a simply connected space such that $\operatorname{dim} H^{p}(Z, \mathbf{Q})<\infty$ for every $p$, and let $L_{Z}$ be a DG-Lie algebra which is a model for $Z$.

Then a model for the trivial fibration $Y \times Z$ equipped with a section corresponding to a constant map is the DG-Lie algebra $A \otimes L_{Z}$ with

$$
\left[a \otimes l, a^{\prime} \otimes l^{\prime}\right]=(-1)^{\operatorname{deg} l \operatorname{deg} a^{\prime}} a a^{\prime} \otimes\left[l, l^{\prime}\right]
$$

and

$$
d(a \otimes l)=d a \otimes l+(-1)^{\operatorname{deg} a} a \otimes d l
$$

Proof. $Y \times Z$ equipped with a section corresponding to a constant map has as a model the DG-algebra $A \otimes \mathcal{C}^{*}\left(L_{Z}\right)$ equipped with the natural augmentation.

REMARK. It follows that a model for the space of maps $Y \rightarrow Z$ homotopic to a constant map is the DG-Lie algebra $\left(A \otimes L_{Z}\right)_{+}$.

(6.2) A connected component of $Z^{S^{n}}$. Let $Z$ be a simply connected space such that $\operatorname{dim} H^{P}(Z, \mathbf{Q})<\infty$ for every $p$. Let $L_{Z}$ be a DG-Lie algebra which is a model for $Z$. Let $f: S^{n} \rightarrow Z$ be a continuous map. There exists a $(n-1)$-cycle $l \in L_{Z}$ such that its homology class $l \in H_{n-1}\left(L_{Z}\right)$ is equal to the rational homotopy class of $f$, for $H_{n-1}\left(L_{Z}\right)=\pi_{n}(Z) \otimes_{\mathbf{Z}} \mathbf{Q}$.

Let $A$ be either the quotient algebra $\mathbf{Q}[a] / a^{2}$ or the free algebra $\Lambda(a)$, respectively if $n=\operatorname{deg} a$ is even or odd.

Then a model for the trivial fibration $S^{n} \times Z$ equipped with the crosssection corresponding to $f$ is the $D G$-Lie-algebra $A \otimes L_{Z}$ with

$$
\left[a \otimes l, a^{\prime} \otimes l^{\prime}\right]=(-1)^{\operatorname{deg} l \operatorname{deg} a^{\prime}} a a^{\prime} \otimes\left[l, l^{\prime}\right]
$$

and, for every $x \in L_{Z}$,

$$
d(1 \otimes x)=1 \otimes d x-a \otimes[l, x] .
$$


In particular, the DG-Lie algebra $\left(A \otimes L_{Z}\right)_{+}$with same bracket and differential is a model for the connected component $\left(Z^{S^{n}}, f\right)$ of $f$.

For instance, with this model one can immediately see that the connected component $\left(\left(S^{2 k}\right)^{S^{2 k}}\right.$, constant map) has the same rational homotopy type as $K(\mathbf{Q}, 2 n-1) \times S^{2 n}$, whereas the connected component $\left(\left(S^{2 k}\right)^{S^{2 k}}\right.$, any map nonhomotopic to a constant map) has the same rational homotopy type as $K(\mathbf{Q}, 4 n-1)$.

Proof. Let us first see that it does not depend on the choice of $l$. If $\tilde{l}$ is a $(n-1)$-cycle such that $l-\tilde{l}=d \hat{l}, \hat{l} \in\left(L_{Z}\right)_{n}$, then $1 \otimes x \mapsto 1 \otimes x-a$ $\otimes[\hat{l}, x]$ is an isomorphism $\psi$ of $A \otimes L_{Z}$ such that $d \psi=\psi d$, where $\tilde{d}(1 \otimes x)=1 \otimes d x-a \otimes[\tilde{l}, x]$ for every $x \in L_{Z}$.

Next, note that if $l=0$, then we are back to example (6.1).

Assume $l \neq 0$ and let $L(l)$ be the free Lie algebra on $l$. The natural Lie algebra map $L(l) \rightarrow L_{Z}$ induces a map $\mathcal{C}^{*}\left(L_{Z}\right) \rightarrow \mathcal{C}^{*}(L(l))$ on the cochain DG-algebras and thus a DG-algebra map $\lambda$ : $\mathcal{C}^{*}\left(L_{Z}\right) \rightarrow A$.

Then the tensor product $A \otimes \mathcal{C}^{*}\left(L_{Z}\right)$ equipped with the DG-algebra map $\sigma: A \otimes \mathcal{C}^{*}\left(L_{Z}\right) \rightarrow A, \sigma(a \otimes \xi)=a \lambda(\xi)$, is a model for $S^{n} \times Z$ equipped with the cross-section $s_{f}: S^{n} \rightarrow S^{n} \times Z, s_{f}(u)=(u, f(u))$. Therefore, it follows from (4.5) that there exists a differential $d_{L}$ on $L=A \otimes L_{Z}$ such that $\bigodot_{A}^{*}(L)$ equipped with the natural augmentation is a model for $\left(S^{n} \times Z, s_{f}\right)$.

Let us compute the differential $d_{f}$ on $\bigodot_{A}^{*}(L)$ on a given $A$-module base. Let $l_{0}, l_{1}, \ldots, l_{p}, \ldots$ be a base of $L_{Z}$ (as a vector space) such that $l_{0}=l$. As in the last section of $\S 4$, set $v_{i}=s l_{i}^{*}$. Then $d_{f}$ is defined by $d_{f}=\varphi^{-1} d \varphi$, where $\varphi: \mathcal{C}_{A}^{*}(L) \rightarrow A \otimes \mathcal{C}^{*}\left(L_{Z}\right)$ is given by $\varphi\left(v_{0}\right)=v_{0}-a$, $\varphi\left(v_{l}\right)=v_{i}, i \neq 0$, and where $d(1 \otimes \xi)=1 \otimes d \xi$ for $\xi \in \mathcal{C}^{*}\left(L_{Z}\right)$.

Now we have (see §4)

$$
d v_{h}=(-1)^{\operatorname{deg} v_{h}}\left(\sum_{i} a_{i}^{h} v_{i}+\frac{1}{2} \sum_{i, j}(-1)^{\operatorname{deg} l_{i}} c_{i j}^{h} v_{i} v_{j}\right),
$$

where

$$
d l_{i}=\sum_{j} a_{i}^{j} l_{j} \quad \text { and } \quad\left[l_{l}, l_{j}\right]=\sum_{k} c_{i j}^{k} l_{k} .
$$

(Note that $a_{0}^{j}=0$ and $a_{i}^{j}, c_{l j}^{k} \in \mathbf{Q}$.) It follows that

$$
\begin{aligned}
d_{f} v_{h} & =\varphi^{-1}\left((-1)^{\operatorname{deg} v_{h}}\left(\sum_{i} a_{t}^{h} v_{i}+\frac{1}{2} \sum_{i, j}(-1)^{\operatorname{deg} l_{i}} c_{i j}^{h} v_{i} v_{j}\right)\right) \\
& =d v_{h}+(-1)^{\operatorname{deg} v_{h}} \frac{1}{2} a \sum_{i}\left((-1)^{\operatorname{deg} v_{l} \operatorname{deg} a+\operatorname{deg} l_{t}} c_{i 0}^{h}+(-1)^{\operatorname{deg} l_{0}} c_{0 i}^{h}\right) v_{i} .
\end{aligned}
$$


But

$$
\begin{aligned}
c_{i 0}^{h} & =v_{h}\left(s\left[l_{i}, l_{0}\right]\right)=-(-1)^{\operatorname{deg} l_{i} \operatorname{deg} l_{0}} v_{h}\left(s\left[l_{0}, l_{i}\right]\right) \\
& =-(-1)^{\operatorname{deg} l_{i} \cdot \operatorname{deg} l_{0}} c_{0 i}^{h}
\end{aligned}
$$

and $\operatorname{deg} l_{j}=\operatorname{deg} v_{j}-1, j \geq 0$. It follows that

$$
d_{f} v_{h}=d v_{h}+(-1)^{\operatorname{deg} v_{h}+\operatorname{deg} l_{0}} \sum_{i} a c_{0 i}^{h} v_{i} .
$$

But this is the differential induced by the differential $\tilde{d}_{f}$ on $L$, given by

$$
\tilde{d}_{f}\left(1 \otimes l_{i}\right)=1 \otimes d l_{i}-a \otimes\left[l_{0}, l_{i}\right] .
$$

Hence $\tilde{d}_{f}=d_{L}$ and this ends the proof.

ReMARK. For every $p \geq 1$, let $\alpha_{p}: H_{p+1}(L) \rightarrow H_{p+n}(L)$ be the map defined by $\alpha_{p}(\bar{x})=\overline{[l, x]}, \beta_{p}: H_{p+n}(L) \rightarrow H_{p}\left((A \otimes L)_{+}\right)$the map defined by $\beta_{p}(\bar{x})=\overline{(a \otimes x)}_{+}$and $\gamma_{p}: H_{p}\left((A \otimes L)_{+}\right) \rightarrow H_{p}(L)$ the map induced in degree $p$ by $\varepsilon \otimes$ id: $A \otimes L \rightarrow L$, where $\varepsilon: A \rightarrow \mathbf{Q}$ is the natural augmentation of $A$. Then one can directly see that the following sequence is exact:

$$
\cdots \rightarrow H_{p+1}(L) \stackrel{\alpha_{p}}{\rightarrow} H_{p+n}(L) \stackrel{\beta_{p}}{\rightarrow} H_{p}\left((A \otimes L)_{+}\right) \stackrel{\gamma_{p}}{\rightarrow} H_{p}(L) \rightarrow \cdots
$$

It is Whitehead's exact sequence for the rational homotopy [4]:

$$
\begin{aligned}
\cdots \pi_{p+2}(Z) \otimes_{\mathbf{Z}} \mathbf{Q} & \rightarrow \pi_{p+n+1}(Z) \otimes_{\mathbf{Z}} \mathbf{Q} \rightarrow \pi_{p+1}\left(Z^{S^{n}}, f\right) \otimes_{\mathbf{Z}} \mathbf{Q} \\
& \rightarrow \pi_{p+1}(Z) \otimes_{\mathbf{Z}} \mathbf{Q} \rightarrow \cdots .
\end{aligned}
$$

(6.3) A connected component of $\left(S^{n}\right)^{Y}$. Let $Y$ be a nilpotent space and let $A$ be a finite dimensional DG-algebra which is a model for $Y$.

Let $n>1$ and $f: Y \rightarrow S^{n}$ be a continuous map. It induces a map $f^{*}$ : $H^{*}\left(S^{n}, \mathbf{Q}\right) \rightarrow H^{*}(Y, \mathbf{Q})$. Let $\xi \in H^{n}\left(S^{n}, \mathbf{Q}\right)$ be a generator and $a \in A^{n}$ be a cocycle such that $f^{*}(\xi)=\bar{a} \in H^{n}(A)=H^{n}(Y, \mathbf{Q})$.

Then the DG-Lie algebra $A \otimes L(l)$ over $A$, with $\operatorname{deg} l=n-1$ and

$$
d(1 \otimes l)=-a \otimes[l, l]
$$

is a model for the trivial fibration $Y \times S^{n}$ equipped with the cross-section $s_{f}$ given by $f$.

It follows, in particular, that $(A \otimes L(l))_{+}$is a model for $\left(\left(S^{n}\right)^{Y}, f\right)$. 
Proof. Let us first see that this does not depend on the choice of $a$. If $a-\tilde{a}=d a_{0}$, then $\psi(1 \otimes l)=1 \otimes l-a_{0} \otimes[l, l]$ and $\psi(1 \otimes[l, l])=1 \otimes$ $[l, l]$ defines an automorphism of $A \otimes L(l)$ such that $\tilde{d} \psi=\psi d$, where $\tilde{d}(1 \otimes l)=-\tilde{a} \otimes[l, l]$.

If $n$ is odd, then $S^{n}=K(\mathbf{Q}, n)$ and $[l, l]=0$, so that (6.3) means $\left(K(\mathbf{Q}, n)^{Y}, f\right)$ has the same rational homotopy type as $\left(K(\mathbf{Q}, n)^{Y}\right.$, ct $=$ constant map). Now, the fact that $\left(K(\mathbf{Q}, n)^{Y}, f\right)$ and $\left(K(\mathbf{Q}, n)^{Y}, \mathrm{ct}\right)$ have the same homotopy type has been shown by Thom [13], and this result is used by Haefliger [8] to prove the theorem we apply to get our Theorem 2. For us, therefore, only the case " $n$ is even" is interesting.

Thus, assume $n$ is even and let $A \otimes e^{*}(L(l))=A(v, w)$, with $\operatorname{deg} v$ $=n, \operatorname{deg} w=2 n-1$ and $d w=\frac{1}{2} v^{2}$. (As in $\S 4$, we have set $v=s l^{*}$ and $w=s[l, l]^{*}$.) As $f^{*}(\xi)=\bar{a}$ and $\xi^{2}=0$, there exists an element $b \in A$ such that $d b=\frac{1}{2} a^{2}$. The cross-section $s_{f}$ corresponds to the DG-algebra map $\sigma_{f}: A(v, w) \rightarrow A$ defined by $\sigma_{f}(v)=a$ and $\sigma_{f}(w)=b$.

From (4.5) it follows that there exists a differential $d_{L}$ on $A \otimes L(l)$ such that $\bigodot_{A}^{*}(L)$ equipped with the natural augmentation is a model for $\left(\left(S^{n}\right)^{Y}, f\right)$. The differential $d_{f}$ on $\bigodot_{A}^{*}(L)$ is given by $d_{f}=\varphi^{-1} d \varphi$, where $\varphi$ : $\mathcal{C}_{A}^{*}(L) \rightarrow A \otimes \mathcal{C}^{*}(L(l))$ is defined by $\varphi(v)=v-a$ and $\varphi(w)=w-b$. Hence, we have $d_{f} v=0$ and $d_{f} w=\frac{1}{2} v^{2}+a v$. This is the differential induced by the differential $d_{L}$ defined on $L$ by $d_{L}(1 \otimes l)=-a \otimes[l, l]$ and $d_{L}(1 \otimes[l, l])=0$, for

$$
d_{f} w(s(1 \otimes l))=(-1)^{\operatorname{deg} w} w(s d(1 \otimes l))=-w(-s(a \otimes[l, l]))=a .
$$

REMARK. Let $\alpha_{p-1}: H^{n-p-1}(A) \rightarrow H^{2 n-p-1}(A)$ be defined by $\alpha_{p-1}\left(\bar{a}^{\prime}\right)=\overline{a a^{\prime}}$, let $\beta_{p-1}: H^{2 n-p-1}(A) \rightarrow H_{p-1}\left((A \otimes L(l))_{+}\right)$be defined by $\beta_{p-1}\left(\bar{a}^{\prime}\right)=\overline{a^{\prime}} \otimes[l, l]$ and let $\gamma_{p-1}: H_{p-1}\left((A \otimes L(l))_{+}\right) \rightarrow H^{n-p}(A)$ be defined in the following way: a $(p-1)$-cycle $\xi$ in $(A \otimes L(l))_{+}$is of the form $a_{0} l+a_{1}[l, l]$, where $a_{0} \in A^{n-p}, a_{1} \in A^{2 n-p-1}$. Let $\gamma_{p-1}(\bar{\xi})=a_{0}$. Then the following sequence is exact:

$$
\begin{aligned}
\cdots H^{n-p-1}(A) \stackrel{\alpha_{p-1}}{\rightarrow} H^{2 n-p-1}(A) \stackrel{\beta_{p-1}}{\rightarrow} H_{p-1}\left((A \otimes L(l))_{+}\right) \\
\stackrel{\gamma_{p-1}}{\rightarrow} H^{n-p}(A) \rightarrow \cdots .
\end{aligned}
$$

It is Federer's exact sequence [4]

$$
\begin{aligned}
\cdots & \rightarrow H^{n-p-1}(Y, \mathbf{Q}) \rightarrow H^{2 n-p-1}(Y, \mathbf{Q}) \rightarrow \pi_{p}\left(\left(S^{n}\right)^{Y}, f\right) \\
& \rightarrow H^{n-p}(Y, \mathbf{Q}) \rightarrow \cdots .
\end{aligned}
$$


(6.4) A fibration over $S^{2}$ defined by a "twisted bracket". Here we give an example of a nontrivial fibration which has as a model a graded Lie algebra (with trivial differential) over $A=H^{*}\left(S^{2}, \mathbf{Q}\right)$ with a "twisted bracket".

Let $a \in H^{*}\left(S^{2}, \mathbf{Q}\right)$ be the fundamental class. Let $L^{\prime}$ be the graded Lie algebra (over $\mathbf{Q}$ ) of dimension 2 as a vector space, with base $l, l^{\prime}$, $\operatorname{deg} l=3, \operatorname{deg} l^{\prime}=8, d l=d l^{\prime}=0,[l, l]=\left[l, l^{\prime}\right]=\left[l^{\prime}, l^{\prime}\right]=0$.

We define on $L=A \otimes L^{\prime}$ a DG-Lie algebra structure over $A$ by $[1 \otimes l, 1 \otimes l]=a \otimes l^{\prime}$ and $\left[a \otimes l^{\prime}, a \otimes l^{\prime}\right]=\left[1 \otimes l, 1 \otimes l^{\prime}\right]=0, d(1 \otimes l)$ $=d\left(1 \otimes l^{\prime}\right)=0$. Then $L$ is a model for a non-trivial fibration over $S^{2}$ whose fibre is $K(\mathbf{Q}, 4) \times K(\mathbf{Q}, 9)$ and whose minimal model over $A$ is given by $A(x, y)$ with $\operatorname{deg} x=4, \operatorname{deg} y=9, d y=-a x^{2}$.

Now, as a Lie algebra, $L=L_{+}$and thus the space of sections of this fibration has the same rational homotopy type as $K(\mathbf{Q}, 2) \times S^{4} \times K(\mathbf{Q}, 9)$ (whereas the space of maps from $S^{2}$ to $K(\mathbf{Q}, 4) \times K(\mathbf{Q}, 9)$ has the same rational homotopy type as $K(\mathbf{Q}, 2) \times K(\mathbf{Q}, 4) \times K(\mathbf{Q}, 7) \times K(\mathbf{Q}, 9))$.

(6.5) A fibration over $S^{3}$ defined by a trivial bracket and a "twisted differential". Here we give an example of a non-trivial fibration which has as a model a DG-Lie algebra over $H^{*}\left(S^{3}, \mathrm{Q}\right)$ with trivial bracket.

Let $S^{15} \rightarrow S^{8}$ be the Hopf fibration over $S^{8}$ whose fibre is $S^{7}$. Let $S^{3} \times S^{5} \rightarrow S^{8}$ a degree-1 map and let $E \rightarrow S^{3} \times S^{5}$ be the induced fibration. Consider the fibration $E \rightarrow S^{3}$ obtained by taking the projection over the first factor. Its fiber is $S^{5} \times S^{7}$.

Let $A=H^{*}\left(S^{3}, \mathbf{Q}\right)$ and let $a \in A$ be the fundamental class. Let $L^{\prime}$ be the trivial Lie algebra with two elements $l, l^{\prime}$ with $\operatorname{deg} l=4$ and $\operatorname{deg} l^{\prime}=6$ (differentials and brackets are trivial). Let $L$ be the DG-Lie algebra over $A$ obtained by equipping the product $A \otimes L^{\prime}$ with the differential defined by $d(1 \otimes l)=a \otimes l^{\prime}$ and $d\left(1 \otimes l^{\prime}\right)=0$. Then $L$ is a model for $E \rightarrow S^{3}$, for $\bigodot_{A}^{*}(L)$ is the free minimal model of $E \rightarrow S^{3}$ over $A$. As $L_{+}=L$ and (also considered over $\mathbf{Q}) L$ contains a trivial sub-Lie algebra which has as a base the element $a \otimes \lambda$ and $1 \otimes \mu$, and as this inclusion is a weak equivalence, the space of sections of $E \rightarrow S^{3}$ has the same rational homotopy type as the product $K(\mathbf{Q}, 2) \times K(\mathbf{Q}, 7)$ of Eilenberg-MacLane complexes.

(6.6) Rational homotopy type of immersions of a $k$-dimensional manifold in $\mathbf{R}^{p}$. Let $X$ be a differentiable manifold of dimension $k$. The space of 
immersions of $X$ in $\mathbf{R}^{p}$ with the $C^{\infty}$-topology has the same weak homotopy type as the space of cross-sections of the bundle $\mathcal{E}$ over $X$ associated to the bundle of $k$-frames of $X$, whose fiber is the Stiefel variety $V_{p, k}$ of $k$-frames in $\mathbf{R}^{p}$ [9].

Let $A$ be a model for $X$. We wish to commpute first the minimal model of $\mathcal{E}$ over $A$. To this purpose we need the following general considerations.

Proposition. Let $G$ be a connected compact Lie group and $E G \rightarrow B G$ the universal $G$-principal bundle. Let $H^{*}(B G, \mathbf{Q})=\mathbf{Q}\left(c_{1}, \ldots, c_{l}\right)$ with $\operatorname{deg} c_{i}$ even, $1 \leq i \leq l$. Then the DG-algebra $\mathbf{Q}\left(c_{1}, \ldots, c_{l}\right) \otimes \mathbf{Q}\left(u_{1}, \ldots, u_{l}\right) \otimes$ $\mathbf{Q}\left(c_{1}^{\prime}, \ldots, c_{l}^{\prime}\right)$, with $\operatorname{deg} c_{l}^{\prime}=\operatorname{deg} c_{i}, \operatorname{deg} u_{\imath}=\operatorname{deg} c_{i}-1, d c_{i}=d c_{i}^{\prime}=0, d u_{i}$ $=c_{l}-c_{l}^{\prime}$, is a model over $\mathbf{Q}\left(c_{1}, \ldots, c_{l}\right)$ for the bundle $p_{1}: E G \times{ }_{G} E G \rightarrow B G$ induced by the projection on the first factor.

Proof. First, consider the two bundles $p_{1}, p_{2}: E G \times{ }_{G} E G \rightarrow B G$ induced, respectively, by the projection on each factor and whose fiber is $E G$. Let $\Delta: B G \rightarrow E G \times{ }_{G} E G$ be the map defined, for $b=e G \in B G$, by $\Delta b=$ the class of $(e, e)$ in $E G \times{ }_{G} E G$. Then $\Delta$ is a cross-section for both $p_{1}$ and $p_{2}$ and it is a homotopy equivalence (for $E G$ is acyclic). In particular, $p_{1}$ and $p_{2}$ are homotopic to each other.

Next, choose a DG-algebra weak equivalence $f: \mathbf{Q}\left(c_{1}, \ldots, c_{l}\right) \rightarrow$ $A^{*}(B G)$. (For any space $Y, A^{*}(Y)$ is the DG-algebra of Q-polynomial differential forms over the singular complex of $Y$.) As $H^{*}\left(p_{1}\right)\left(c_{l}\right)=$ $H^{*}\left(p_{2}\right)\left(c_{l}\right)$, there exists a form $w_{l} \in A^{*}\left(E G \times{ }_{G} E G\right)$ such that $d w_{i}=$ $A^{*}\left(p_{1}\right)\left(f c_{i}\right)-A^{*}\left(p_{2}\right)\left(f c_{i}\right)$, where $A^{*}\left(p_{j}\right): A^{*}(B G) \rightarrow A^{*}\left(E G \times{ }_{G} E G\right)$, $j=1,2$, are the DG-maps induced on the differential forms. Then the DG-algebra map

$$
g: \mathbf{Q}\left(c_{1}, \ldots, c_{l}\right) \otimes \mathbf{Q}\left(u_{1}, \ldots, u_{l}\right) \otimes \mathbf{Q}\left(c_{1}^{\prime}, \ldots, c_{l}^{\prime}\right) \rightarrow A^{*}\left(E G \times_{G} E G\right)
$$

defined by $g\left(c_{i}\right)=A^{*}\left(p_{1}\right)\left(f c_{i}\right), \quad g\left(u_{i}\right)=w_{l}$ and $g\left(c_{i}^{\prime}\right)=A^{*}\left(p_{2}\right)\left(f c_{l}\right), 1$ $\leq i \leq l$, is a weak-equivalence. This can be proved by considering the commutative diagram

$$
\begin{aligned}
\mathbf{Q}\left(c_{1}, \ldots, c_{l}\right) \otimes \mathbf{Q}\left(u_{1}, \ldots, u_{l}\right) \otimes \mathbf{Q}\left(c_{1}^{\prime}, \ldots, c_{l}^{\prime}\right) \stackrel{g}{\longrightarrow} & A^{*}\left(E G \times_{G} E G\right) \\
\mathbf{\nu} \uparrow & \uparrow A^{*}\left(p_{1}\right)
\end{aligned}
$$


where $\nu\left(c_{i}\right)=c_{i}$, and showing that $\nu$ is a weak-equivalence, which can be done as follows. Set

$$
\pi: \mathbf{Q}\left(c_{1}, \ldots, c_{l}\right) \otimes \mathbf{Q}\left(u_{1}, \ldots, u_{l}\right) \otimes \mathbf{Q}\left(c_{1}^{\prime}, \ldots, c_{l}^{\prime}\right) \rightarrow \mathbf{Q}\left(c_{1}, \ldots, c_{l}\right),
$$

$\pi\left(c_{l}\right)=c_{i}, \pi\left(c_{i}^{\prime}\right)=c_{l}, \pi\left(u_{l}\right)=0$. Then $\pi \nu=$ identity and $\nu \pi$ is homotopic to the identity, with the homotopy $H$ defined by $H c_{i}=c_{i}, H u_{i}=u_{i} t$ and $H c_{i}^{\prime}=c_{l}(1-t)+c^{\prime} t+u_{i} d t$.

COROLlary. Let $U \rightarrow Y$ be a G-principal bundle and $\alpha: Y \rightarrow B G$ a classifying map. Let $B$ be a model for $Y$ and $\rho: Q\left(c_{1}, \ldots, c_{l}\right) \rightarrow B a$ $D G$-algebra map corresponding to $\alpha$. Then the DG-algebra $\mathbf{Q}\left(c_{1}^{\prime}, \ldots, c_{l}^{\prime}\right) \otimes$ $\mathbf{Q}\left(u_{1}, \ldots, u_{l}\right) \otimes B$ with $d u_{l}=\rho\left(c_{i}\right)-c_{l}^{\prime}$ is a model over $\mathbf{Q}\left(c_{1}^{\prime}, \ldots, c_{l}^{\prime}\right)$ for the bundle $\beta: E G \times{ }_{G} U \rightarrow Y$ induced by $U \rightarrow Y$.

Proof. Let $\hat{\alpha}: U \rightarrow E G$ be the map induced by $\alpha$ and consider the commutative diagram

$$
\begin{array}{ccc}
E G \times{ }_{G} U & \stackrel{\tilde{\alpha}}{\rightarrow} & E G \times{ }_{G} E G \\
\beta \downarrow & & \downarrow p_{1} \\
Y & \vec{\alpha} & B G
\end{array}
$$

where $\tilde{\alpha}$ is induced by id $\times \hat{\alpha}$. (Note that $\beta$ is a homotopy equivalence, for $E G$ is acyclic.) Since $p_{1}$ and $p_{2}$ are homotopic, $\alpha \beta=p_{1} \tilde{\alpha}$ is also homotopic to $p_{2} \tilde{\alpha}$. Now, it follows from the proposition that $B \otimes \mathbf{Q}\left(u_{1}, \ldots, u_{l}\right) \otimes$ $\mathbf{Q}\left(c_{1}^{\prime}, \ldots, c_{l}^{\prime}\right)$, with $d u_{l}=\rho\left(c_{l}\right)-c_{i}^{\prime}$, is a model for $E G \times{ }_{G} U$ over $B$, hence $\mathbf{Q}\left(c_{1}^{\prime}, \ldots, c_{l}^{\prime}\right) \otimes \mathbf{Q}\left(u_{1}, \ldots, u_{l}\right) \otimes B$, with $d u_{l}=\rho\left(c_{i}\right)-c_{l}^{\prime}$, is a model for $E G \times{ }_{G} U \rightarrow B G$ over $\mathbf{Q}\left(c_{1}^{\prime}, \ldots, c_{l}^{\prime}\right)$.

Now, let us return to our bundle $\mathcal{E} \rightarrow X$ whose fiber is $V_{p, k}$. The group $\mathrm{SO}_{k}$ acts naturally on the right on $V_{p, k}$ and we have a $\mathrm{SO}_{k}$-principal bundle $V_{p, k} \rightarrow G_{p, k}$ induced by this action, where $G_{p, k}$ is the Grassmann variety of $k$-planes in $\mathbf{R}^{p}$. Consider this action on $V_{p, k}$. Then $\mathscr{E} \rightarrow X$ is induced from the bundle $E \mathrm{SO}_{k} \times_{\mathrm{SO}_{k}} V_{p, k} \rightarrow B \mathrm{SO}_{k}$ by a map $X \rightarrow B \mathrm{SO}_{k}$ which classifies the tangent bundle over $X$ :

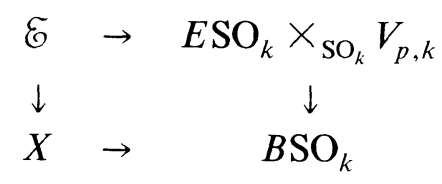

Let $p=2 n$ and $k=2 m, m<n$. (The computations with both $k$ and $p$ odd or with one of the two odd are analogous to those we shall make.) 
Then $H^{*}\left(B \mathrm{BO}_{2 m}, \mathbf{Q}\right)=\mathbf{Q}\left(p_{1}, \ldots, p_{m-1}, \chi\right), \operatorname{deg} p_{i}=4 i, \operatorname{deg} \chi=2 m$. By the above corollary we have

$$
\mathbf{Q}\left(p_{1}, \ldots, p_{m-1}, \chi\right) \otimes \mathbf{Q}\left(u_{1}, \ldots, u_{m-1}, z\right) \otimes B,
$$

with

$$
d u_{i}=\rho\left(p_{i}\right)-p_{i} \text { and } d z=\rho(\chi),
$$

where $B$ is a model for $G_{2 n, 2 m}$ and $\rho: \mathbf{Q}\left(p_{1}, \ldots, p_{m-1}, \chi\right) \rightarrow B$ corresponds to a classifying map $G_{2 n, 2 m} \rightarrow B \mathrm{SO}_{2 m}$ (for example the natural inclusion) is a model for $E \mathrm{SO}_{2 m} \times{ }_{\mathrm{SO}_{2 m}} V_{2 n, 2 m}$ over $\mathbf{Q}\left(p_{1}, \ldots, p_{m-1}, \chi\right)$.

$A$ model for the Grassmann variety $G_{2 n, 2 m}$ of $2 m$-planes in $\mathbf{R}^{2 n}$. Let $H^{*}\left(B \mathrm{SO}_{2 m}, \mathbf{Q}\right)=\mathbf{Q}\left(p_{1}^{\prime}, \ldots, p_{m-1}^{\prime}, \chi^{\prime}\right), \quad \operatorname{deg} p_{i}^{\prime}=4 i, \quad \operatorname{deg} \chi^{\prime}=2 m$, and

$$
\begin{gathered}
H^{*}\left(B \mathrm{SO}_{2(n-m)}, \mathbf{Q}\right)=\mathbf{Q}\left(p_{1}^{\prime \prime}, \ldots, p_{n-m-1}^{\prime \prime}, \chi^{\prime \prime}\right), \\
\operatorname{deg} p_{i}^{\prime \prime}=4 i, \quad \operatorname{deg} \chi^{\prime \prime}=2(n-m) .
\end{gathered}
$$

Then the DG-algebra

$$
B=\mathbf{Q}\left(p_{1}^{\prime}, \ldots, p_{m-1}^{\prime}, \chi^{\prime}\right) \otimes \mathbf{Q}\left(p_{1}^{\prime \prime}, \ldots, p_{n-m-1}^{\prime \prime}, \chi^{\prime \prime}\right) \otimes \mathbf{Q}\left(v_{1}, \ldots, v_{n-1}, w\right)
$$

with

$$
d p_{1}^{\prime}=d \chi^{\prime}=d p_{i}^{\prime \prime}=d \chi^{\prime \prime}=0, \quad d v_{i}=\sum_{l=0}^{i} p_{l}^{\prime} p_{i-l}^{\prime \prime}
$$

and

$$
d w=\chi^{\prime} \chi^{\prime \prime}
$$

(where $p_{0}=p_{0}^{\prime}=1, p_{m}^{\prime}=\frac{1}{2} \chi^{\prime 2}, p_{n-m}^{\prime}=\frac{1}{2} \chi^{\prime \prime 2}, p_{l}^{\prime}=0$ if $l>m, p_{l}^{\prime \prime}=0$ if $l>n-m)$ is a model for $G_{2 n, 2 m}$.

This can be proved by showing directly that the DG-algebra

$$
\mathbf{Q}\left(p_{1}, \ldots, p_{n-1}, \chi\right) \otimes B
$$

with

$$
\begin{gathered}
d p_{i}=d \chi=d p_{i}^{\prime}=d \chi^{\prime}=d p_{i}^{\prime \prime}=d \chi^{\prime \prime}=0, \\
d v_{i}=\sum_{l=0}^{i} p_{l}^{\prime} p_{i-l}^{\prime \prime}-p_{l},
\end{gathered}
$$

and

$$
d w=\chi^{\prime} \chi^{\prime \prime}-\chi
$$


(with the same conventions as above) is a model over $H^{*}\left(B \mathrm{SO}_{2 n}, \mathbf{Q}\right)=$ $\mathbf{Q}\left(p_{1}, \ldots, p_{n-1}, \chi\right)$ for the bundle $B \mathrm{SO}_{2 n} \times B \mathrm{SO}_{2(n-m)} \rightarrow B \mathrm{SO}_{2 n}$ whose fiber is $G_{2 n, 2 m}$.

The minimal model of $V_{2 n, 2 m}$. Let $B$ be as above. To the natural inclusion $G_{2 n, 2 m} \rightarrow B \mathrm{SO}_{2 m}$ corresponds the natural DG-algebra map $\mathbf{Q}\left(p_{1}^{\prime}, \ldots, p_{m-1}^{\prime}, \chi^{\prime}\right) \rightarrow B$. Hence

$$
\mathbf{Q}\left(u_{1}, \ldots, u_{m-1}, z\right) \otimes B
$$

with

$$
d u_{l}=p_{l}^{\prime}, d z=\chi^{\prime} \quad \text { and } \quad d v_{l}=\sum_{l=0}^{i} p_{l}^{\prime} p_{i-l}^{\prime \prime}
$$

(with the same conventions as above) is a model for $V_{2 n, 2 m}$.

Set

$$
\Re=\mathbf{Q}\left(\chi^{\prime \prime}, v_{n-m}, v_{n-m+1}, \ldots, v_{n-1}, w\right)
$$

with

$$
d \chi^{\prime \prime}=0, \quad d v_{n-m}=\frac{1}{2} \chi^{\prime \prime 2} \quad \text { and } \quad d v_{l}=d w=0, \quad i>n-m .
$$

Then it is easy to verify that the natural projection $\mathbf{Q}\left(u_{1}, \ldots, u_{m-1}, z\right)$ $\otimes B \rightarrow \Re$ is a weak equivalence.

Thus $\mathfrak{T}$ is a model for $V_{2 n, 2 m}$, namely its minimal model.

The minimal model of $\mathcal{E} \rightarrow X$ for $k=2 m, p=2 n$. Consider the product $\mathbf{Q}\left(p_{1}, \ldots, p_{m-1}, \chi\right) \otimes \Re$ with $d v_{n-m}=\frac{1}{2} \chi^{\prime \prime 2}, d v_{l}=-p_{i}$ and $d w$ $=-\chi \chi \chi^{\prime \prime}$. Then the above projection $\mathbf{Q}\left(u_{1}, \ldots, u_{n-1}, z\right) \otimes B \rightarrow$ ๆ induces a projection

$$
\mathbf{Q}\left(p_{1}, \ldots, p_{m-1}, \chi\right) \otimes \mathbf{Q}\left(u_{1}, \ldots, u_{n-1}, z\right) \otimes B \rightarrow \mathbf{Q}\left(p_{1}, \ldots, p_{m-1}, \chi\right) \otimes \Re
$$

which is also a weak-equivalence. It follows that $\mathbf{Q}\left(p_{1}, \ldots, p_{m-1}, \chi\right) \otimes \Re$ is the minimal model of $E \mathrm{SO}_{2 m} \times_{\mathrm{SO}_{2 m}} V_{2 n, 2 m}$ over $\mathbf{Q}\left(p_{1}, \ldots, p_{m-1}, \chi\right)$ and the next proposition follows immediately.

Proposition. Let $A$ be a model for $X$ and $\psi: \mathbf{Q}\left(p_{1}, \ldots, p_{m-1}, \chi\right) \rightarrow A a$ map corresponding to a classifying map $X \rightarrow B \mathrm{SO}_{2 m}$ for the tangent bundle on $X$. Then $\Re_{\tilde{G}}=A \otimes \Re$, where $\Re=\mathbf{Q}\left(\chi^{\prime \prime}, v_{n-m}, v_{n-m+1}, \ldots, v_{n-1}, w\right)$, $\operatorname{deg} \chi^{\prime \prime}=2(n-m), \quad \operatorname{deg} v_{1}=4 i-1, \quad \operatorname{deg} w=2 n-1$, with $d \chi^{\prime \prime}=0$, $d v_{n-m}=\frac{1}{2} \chi^{\prime \prime 2}, d v_{l}=-\psi\left(p_{l}\right), i>n-m$, and $d w=-\psi(\chi) \chi^{\prime \prime}$ is the minimal model of $\mathcal{E}$ over $A$. 
A Model for the Space of Immersions $X \rightarrow \mathbf{R}^{2 n}$ Homotopic to a GIVEN ONE.

According to Proposition (4.4), if $\psi\left(p_{i}\right) \neq 0$ for a given $i=1, \ldots$, $m-1$, then $\Re_{\tilde{E}}$ is not the DG-algebra of cochains on a DG-Lie algebra. Let $\sigma: \Re_{\mathscr{E}} \rightarrow A$ be an augmentation on $\Re_{\mathscr{E}}$ and set $\Re_{\mathscr{E}}^{\prime}$ for $\Re_{\mathscr{E}}$ equipped with the differential $d^{\prime}$ defined by $d^{\prime} \chi^{\prime \prime}=0, d^{\prime} v_{n-m}=\frac{1}{2} \chi^{\prime \prime 2}+$ $\sigma\left(\chi^{\prime \prime}\right) \chi^{\prime \prime}, d v_{i}=0, d^{\prime} w=-\psi(\chi) \chi^{\prime \prime}$. Then the $A$-algebra map $\varphi: \Re_{\tilde{E}}^{\prime} \rightarrow$ $\Re_{\mathscr{E}}$ defined by $\varphi\left(\chi^{\prime \prime}\right)=\chi^{\prime \prime}-\sigma\left(\chi^{\prime \prime}\right), \varphi\left(v_{i}\right)=v_{\imath}-\sigma\left(v_{l}\right)$ and $\varphi(w)=$ $w-\sigma(w)$ is a DG-algebra isomorphism over $A$, as can be checked directly. From (4.4) and computations analogous to those in (6.2) or (6.3) it follows that $\Re_{\mathscr{G}}^{\prime}=\mathcal{C}_{A}^{*}(L)$, where

$$
L=A \otimes\left(L(l) \oplus L\left(l_{n-m+1}\right) \oplus \cdots \oplus L\left(l_{n-1}\right) \oplus L(k)\right),
$$

with $\operatorname{deg} l=2(n-m)-1, \operatorname{deg} l_{i}=4 i-2, \operatorname{deg} k=2 n-2$ and $d l=$ $\psi(\chi) k-\sigma\left(\chi^{\prime \prime}\right)[l, l], d l_{i}=d k=0$. (In $\Re_{\mathfrak{E}}$ we have $\chi^{\prime \prime}=s l^{*}, v_{n-m}=$ $s[l, l]^{*}, v_{l}=s l_{l}^{*}, i>n-m, w=s k^{*}$.)

In particular, the DG-Lie algebra $L_{+}$is a model for the space of immersions $X \rightarrow \mathbf{R}^{2 n}$ homotopic to an immersion $s$ to which corresponds the augmentation $\sigma$.

REMARK. One can easily verify that $\sigma\left(\chi^{\prime \prime}\right)$ is the Euler class of the normal bundle to the immersion $s$. We see therefore that the rational homotopy type of the connected component of $s$ in the space of immersions $X \rightarrow \mathbf{R}^{2 n}$ is completely determined by the Euler class of the normal bundle to $s$.

Immersions of $S^{2 m}$ in $\mathbf{R}^{2 n}$. Let $X$ be $S^{2 m}$. Then to $S^{2 m} \rightarrow B \mathrm{SO}_{2 m}$ corresponds $\mathbf{Q}\left(p_{1}, \ldots, p_{m-1}, \chi\right) \rightarrow A=\mathbf{Q}(a) /\left(a^{2}\right)$ defined by $p_{l} \mapsto 0$ and $\chi \mapsto a$. Let $\sigma: \mathfrak{N}_{\mathscr{E}} \rightarrow \mathbf{Q}(a) /\left(a^{2}\right)$ be an augmentation. Then, as we saw, the DG-Lie algebra

$$
L=\mathbf{Q}(a) /\left(a^{2}\right) \otimes\left(L(l) \oplus L\left(l_{n-m+1}\right) \oplus \cdots \oplus L\left(l_{n-1}\right) \oplus L(k)\right),
$$

over $\mathbf{Q}(a) /\left(a^{2}\right)$, with $d l=a k-\sigma\left(\chi^{\prime \prime}\right)[l, l]$ and $d l_{l}=d k=0$, is a model for $\mathcal{E}$ equipped with a cross-section $s$ corresponding to $\sigma$. But, if $n \neq 2 m$, $\sigma$ is necessarily the trivial augmentation (as there is no generator in degree $2 m$ of $\Re)$, thus $\sigma\left(\chi^{\prime \prime}\right)=0$. If $n=2 m$, then all the augmentations are of the form $\sigma\left(\chi^{\prime \prime}\right)=r a, r \in \mathbf{Q}, \sigma\left(v_{i}\right)=\sigma(w)=0$.

It follows that $L_{+}$with $d l=a k$ if $n \neq 2 m$, with $d l=a k-r a[l, l]$ if $n=2 m$, and with $d l_{i}=d k=0$ in both cases, is a model for the space of immersions $S^{2 m} \rightarrow \mathbf{R}^{2 n}$ homotopic to $s$. 
If $n \neq 2 m$, let $q$ be first positive integer in the sequence

$$
\begin{aligned}
2(n-m)- & 1-2 m<4(n-m)-2-2 m \\
& <4(n-m+1)-2-2 m<\cdots<4(n-1)-2-2 m .
\end{aligned}
$$

Then, there is a natural injection

$$
\begin{aligned}
& L\left(l_{q}^{\prime}\right) \oplus \cdots \oplus L\left(l_{4(n-1)-2-2 m}^{\prime}\right) \oplus L(\tilde{l}) \oplus L\left(l_{n-m+1}\right) \\
& \oplus \cdots \oplus L\left(l_{n-1}\right) \oplus L(k) \rightarrow L_{+},
\end{aligned}
$$

where $\operatorname{deg} l_{i}^{\prime}=i, \operatorname{deg} l=4(n-m)-2, \operatorname{deg} l_{i}=4 i-2, \operatorname{deg} k=2 n-2$, which is a weak equivalence.

Therefore, we see that a connected component of the space of immersions of $S^{2 m}$ in $\mathbf{R}^{2 n}, n \neq 2 m$, has the same rational homotopy type as

$$
\begin{aligned}
K(\mathbf{Q}, q) & \times \cdots \times \\
\times & \times K(\mathbf{Q}, 4(n-1)-2-2 m) \\
& \quad \times \cdots \times K(\mathbf{Q}, 4(n-1)-2) \times K(\mathbf{Q}, 2 n-2) .
\end{aligned}
$$

If $n=2 m$, then for every $r \in \mathbf{Q}$, there is a natural injection

$$
\begin{aligned}
& L\left(l_{m+1}^{\prime}\right) \oplus \cdots \oplus L\left(l_{2 m-1}^{\prime}\right) \oplus L\left(k^{\prime}\right) \oplus L\left(l_{m+1}\right) \\
& \oplus \cdots \oplus L\left(l_{2 m-1}\right) \oplus L(k) \rightarrow L_{+},
\end{aligned}
$$

where $\operatorname{deg} k^{\prime}=2 m-2, \operatorname{deg} l_{i}^{\prime}=4 i-2 m-2, \operatorname{deg} k=4 m-2, \operatorname{deg} l_{i}=$ $4 i-2$, which is a weak equivalence.

We see that a connected component of the space of immersions $S^{2 m} \rightarrow$ $\mathbf{R}^{4 m}$ has the same rational homotopy type as

$$
\begin{array}{r}
K(\mathbf{Q}, 2 m+2) \times \cdots \times K(\mathbf{Q}, 6 m+6) \\
\times K(\mathbf{Q}, 2 m-2) \times K(\mathbf{Q}, 4 m+2) \\
\times \cdots K(\mathbf{Q}, 8 m-6) \times K(\mathbf{Q}, 4 m-2) .
\end{array}
$$

One can make similar computations for $X$ a product of spheres or a complex projective space and see that also in those cases a connected component of the space of immersions $X \rightarrow \mathbf{R}^{2 n}$ has the same rational homotopy type as a product of Eilenberg-MacLane complexes.

\section{REFERENCES}

[1] M. Bourbaki, Algèbre, Hermann, Paris, 1970.

[2] H. Cartan, Séminaire Cartan, Exposé 3, E.N.S. $7^{\mathrm{e}}$ année, 154/55.

[3] Deligne, Griffiths, Morgan and Sullivan, The real homotopy of Käehler manifolds, Inv. Math., 29 (1975), 245-274.

[4] H. Federer, A study of function spaces by spectral sequences, Trans. Amer. Math. Soc., 82 (1956), 340-361. 
[5] P.-P. Grivel, Suite spectrale et modèle minimal d' une fibration, Thèse, Genève, 1977.

[6] A. Haefliger, Sur la cohomologie de l'algèbre de Lie des champs de vecteurs, Ann. Scient. E.N.S. $4^{\mathrm{e}}$ année, t.9, (1976), 503-532.

[7] _ On the Gelfand-Fuks Cohomology, L'Enseignement mathématique, T. XXIV, fasc. 1-2, (1978), 143-160.

[8] __ Rational homotopy of the space of sections of a nilpotent bundle, preprint.

[9] M. W. Hirsch, Immersions of manifolds, Trans. Amer. Math. Soc., 93 (1959), 242-276.

[10] J. Neisendorfer, Lie algebras, coalgebras and rational homotopy theory for nilpotent spaces, Pacific J. Math., 74 (1978), 429-460.

[11] D. Quillen, Rational homotopy theory, Amer. Ann. of Math., 90 (1969), 205-295.

[12] D. Sullivan, Infinitesimal computations in topology, IHES, Publ. Math., No. 47, (1977), 269-331.

[13] R. Thom, L'homologie des espaces fonctionnels, Colloque de Topologie algébrique, GBRM, Louvain, (1956), 29-39.

Received June 23, 1980. This work was done under the guidance of Professor André Haefliger who has generously given me advice and encouragement. I wish to express my gratitude to him.

UNIVERSITE DE GENEVE

FACULTE DES SCIENCES

Section de Mathematiques

2-4, RUE DU LIËVRE

1211-Geneve 24, SwitzerLAND 


\section{PACIFIC JOURNAL OF MATHEMATICS \\ EDITORS}

Donald BABBITT (Managing Editor)

University of California

Los Angeles, CA 90024

Hugo Rossi

University of Utah

Salt Lake City, UT 84112

C. C. Moore and Arthur Ogus

University of California

Berkeley, CA 94720
J. DugunduI

Department of Mathematics

University of Southern California

Los Angeles, CA 90089-1113

R. FinN and H. SAMELSON

Stanford University

Stanford, CA 94305

ASSOCIATE EDITORS

R. ARENS

E. F. BECKENBACH

B. H. NeUMANN

F. WOLF

K. YosHIDA (1906-1982)

\section{SUPPORTING INSTITUTIONS}

UNIVERSITY OF ARIZONA

UNIVERSITY OF BRITISH COLUMBIA

CALIFORNIA INSTITUTE OF TECHNOLOGY

UNIVERSITY OF CALIFORNIA

MONTANA STATE UNIVERSITY

UNIVERSITY OF NEVADA, RENO

NEW MEXICO STATE UNIVERSITY

OREGON STATE UNIVERSITY
UNIVERSITY OF OREGON

UNIVERSITY OF SOUTHERN CALIFORNIA

STANFORD UNIVERSITY

UNIVERSITY OF HAWAII

UNIVERSITY OF TOKYO

UNIVERSITY OF UTAH

WASHINGTON STATE UNIVERSITY

UNIVERSITY OF WASHINGTON 


\section{Pacific Journal of Mathematics}

\section{Vol. 113, No. $1 \quad$ March, 1984}

Flavio E. A. da Silveira, Rational homotopy theory of fibrations $\ldots \ldots \ldots \ldots 1$

Donald M. Davis, Desuspensions of stunted projective spaces ............ 35

Lou van den Dries, Exponential rings, exponential polynomials and

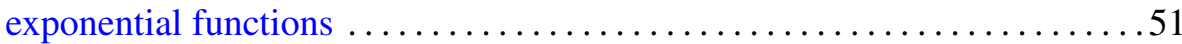

Fred Galvin and Samuel David Shore, Completeness in semimetric

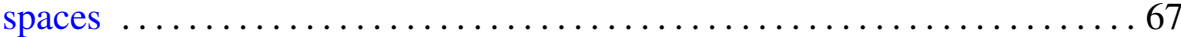

Fereidoun Ghahramani, Compact elements of weighted group algebras . . . 777

Munehiko Itōo, The closed image of a hereditary $M_{1}$-space is $M_{1} \ldots \ldots \ldots 85$

Elvira Laura Livorni, Classification of algebraic surfaces with sectional genus less than or equal to six. I. Rational surfaces .............. 93

H. Alan MacLean, Riesz sets and a theorem of Bochner ............ 115

E. Neher, Jordan triple systems with completely reducible derivation or

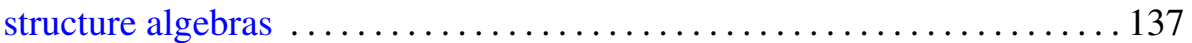

Joe Repka, Shalika's germs for $p$-adic GL( $n)$. I. The leading term $\ldots \ldots \ldots 165$

Joe Repka, Shalika's germs for $p$-adic GL( $n)$. II. The subregular term $\ldots \ldots 173$

Rae Michael Andrew Shortt, Borel density, the marginal problem and isomorphism types of analytic sets $\ldots \ldots \ldots \ldots \ldots \ldots \ldots \ldots \ldots \ldots \ldots \ldots \ldots$

Baruch Solel, The multiplicity functions of invariant subspaces for nonselfadjoint crossed products $\ldots \ldots \ldots \ldots \ldots \ldots \ldots \ldots \ldots \ldots \ldots \ldots . \ldots \ldots$

Su-win Yang, Self-intersection number of immersions and enumeration of nonstable vector bundles

W. M. Zajączkowski, Local solvability of nonstationary leakage problem for ideal incompressible fluid. II 